Décadrages Décadrages

cınéma, à travers champs Cinéma, à travers champs

39 | 2018

Jeu vidéo et cinéma

Les codes du western à l'ère du codage informatique : le jeu vidéo sur la piste du gunfighter cinématographique (Red Dead Redemption)

Alain Boillat

\title{
CpenEdition
}

Journals

Édition électronique

URL : https://journals.openedition.org/decadrages/1340

DOI : $10.4000 /$ decadrages. 1340

ISSN : 2297-5977

Éditeur

Association Décadrages

Édition imprimée

Date de publication : 1 décembre 2018

Pagination : 62-92

ISBN : 978-2-9700963-5-1

ISSN : 2235-7823

Référence électronique

Alain Boillat, « Les codes du western à l'ère du codage informatique : le jeu vidéo sur la piste du gunfighter cinématographique (Red Dead Redemption) », Décadrages [En ligne], 39 | 2018, mis en ligne le 05 février 2021, consulté le 04 avril 2022. URL : http://journals.openedition.org/decadrages/1340 ; DOI : https://doi.org/10.4000/decadrages.1340 
Alain Boillat

\section{Les codes du western à l'ère du codage informatique: le jeu vidéo sur la piste du gunfighter cinématographique (Red Dead Redemption)}

1 Voir Kristen Whissel, «Placing the Spectator on the Scene of History: the Battle Re-enactment at the Turn of the Century, from Buffalo Bill's Wild West to the Early Cinema», Historical Journal of Film, Radio and Television, vol. 22, $\mathrm{n}^{\circ} 3$, 2002, pp. 225-243; Paul Bleton, Western, France. La place de l'Ouest dans l'imaginaire français, Paris, Editions Belles Lettres, 2002.

2 Voir Jean-Louis Leutrat, L'Alliance brisée, Lyon, PUL, I986.

3 Jean-Louis Rieupeyrout, Le Western ou le cinéma américain par excellence, Paris, Edition du Cerf, I953. La préface d'André Bazin a été reprise dans les éditions des écrits de ce dernier, sous le même titre (Qu'est-ce que le cinéma? Vol.3, Cinéma et sociologie, Paris, Cerf, I96I, Pp. I35-I45).

4 Outre les séries au long court telles que Lucky Luke (dès 1947), Tex (dès I948), Jerry Spring (1955-1990), Blueberry (dès I963), Buddy Longway (I974-2006), Comanche (1972-1983), Mac Coy (1974-1999) ou Durango (dès I98I, clairement inspirée des westerns italiens), on peut mentionner les séries plus récentes telles que Bouncer (dès 200I), W.E.S.T. (dès 2003) et Undertaker (dès 2015). Notons par ailleurs que le western a également été revisité récemment dans le domaine de la bande dessinée d'auteur (la série Gus de Christophe Blain, Calamity de Sylvie

HÉRITIER DEs dime novels et des Wild West Shows ${ }^{1}$ - pratiques populaires de la fin du XIX ${ }^{\mathrm{e}}$ siècle qui exacerbent autant la narration à travers des récits feuilletonesques palpitants que l'attraction grâce à des mises en scène spectaculaires -, le western cinématographique s'institutionnalise au cours des années I9IO-I920 2 et s'impose comme un genre majeur de l'industrie hollywoodienne à partir de la toute fin des années i930. Au milieu des années I950, le western a pu ainsi être considéré en France comme «le cinéma américain par excellence ${ }^{3}$, la mythologie de l'Ouest et l'époque des pionniers faisant office d'ancrage à la fois géographique, historique, culturel et symbolique.

Durant la seconde moitié des années i960, l'anachronisme de l'idéologie dominante du western dans un contexte de revendications sociales (défense des minorités, émancipation des femmes, mouvement d'opposition à la guerre du Vietnam) semble avoir contribué, en sus de la réorganisation qui affecte par ailleurs l'ensemble du système de production, à la disparition progressive du genre, désormais détrôné par la sciencefiction qui substitue à la conquête de l'Ouest sauvage la découverte des confins de l'espace intersidéral. Le western fait alors l'objet d'une réappropriation européenne, dans le sillage du succès international des films de Sergio Leone. Alors que le genre s'est maintenu avec une grande constance en Italie et en France dans des séries de bandes dessinées ${ }^{4}$, sans doute parce que ce secteur éditorial est fortement structuré en fonction de genres populaires et se prête à une mise en scène haute en couleur de stéréotypes, on observe inversement qu'au cinéma, les «cowboys» ont

Fontaine, Texas Cowboys de Bonhomme et Trondheim, Marta Jane Cannary de Christian Perrissin et Mathieu Blanchin,
L'Odeur des garçons affamés dessiné par Frederik Peeters, etc.). 
presque totalement déserté le grand écran depuis la fin des années $1970^{5}$, au moment où, précisément, le médium du jeu vidéo sur lequel portera la présente étude devient accessible au grand public grâce au marché des consoles domestiques.

Parallèlement à quelques productions cinématographiques ponctuelles qui ont garanti une persistance du western dans les salles au début du $\mathrm{XXI}^{\mathrm{e}}$ siècle $^{6}$, on notera une résurgence du genre dans le domaine télévisuel, en particulier avec les trois saisons de la série Deadwood diffusée entre 2004 et 2006 par $\mathrm{HBO}^{7}$ et la série Westworld (dès 20ı6), qui, reprenant le motif du parc à thème peuplé d'automates à apparence humaine du film de science-fiction homonyme de $1973^{8}$, met en scène la restauration factice, sous la houlette d'une figure démiurgique de créateur de monde(s), à la fois du passé mythique des Etats-Unis et du genre cinématographique. Or, dans cette série récente, la répétition des actions programmées s'émancipe largement d'un climat fantastique tel qu’on le trouve dans le roman d'Adolfo Bioy Casares, L'Invention de Morel (1950), pour imposer des références explicites à l'expérience du consommateur de jeux vidéo de type RPG (Role Playing Game) ${ }^{9}$, par exemple lorsque l'un des protagonistes principaux éconduit à plusieurs reprises un individu qui se comporte en «personnage non-joueur» en appelant avec insistance une interaction susceptible d'embrayer sur une quête secondaire. L'enjeu principal de la première saison de la série, dans laquelle la frontière entre l'humain et son simulacre s'estompe progressivement, réside d'ailleurs dans la suspension du reboot perpétuel d'activités inlassablement reconduites par les «avatars» à travers des déclinaisons paradigmatiques: il s'agit, pour les androïdes de la série, de s'extraire des boucles itératives régies à la fois par les codes du genre western et par ceux du jeu vidéo. Par conséquent, le régime d'expérience de type ludique (simulant un genre cinématographique) se voit infléchi vers la logique du récit filmique, qui implique quant à elle une chronologie et une construction évolutive de la psychologie des personnages. Si Westworld peut ainsi entrelacer, autour de la problématique réflexive de la création d'un «environnement», les références au cinéma et au jeu vidéo sous l'égide du western, c'est sans doute parce que ce genre, dont l'industrie vidéoludique

5 Exception faite des films de Clint Eastwood et Kevin Costner, réalisateurs/ acteurs/producteurs. Leur obstination à reconduire le genre ne s'inscrit toute- fois pas dans une tendance de l'industrie cinématographique: tout spectateur reconnaît les propriétés du genre, mais aucun ensemble conséquent de films sortis en salles n'en relève plus spécifiquement.

6 On pense notamment à The Proposition (John Hillcoat, Australie/RoyaumeUni, 2005), aux remakes 3: Io to Yuma (James Mangold, Etats-Unis, 2007) et True Grit (Ethan et Joel Cohen, E.-U., 20Io), ou encore à Blackthorn (Mateo Gil, Espagne/F./Bolivie/Grande-Bretagne, 20II), The Salvation (Kristian Levring, Danemark/R.-U./Afrique du Sud/Suède/ Belgique, 20I4), The Hateful Eight (Quentin Tarantino, E.-U., 2015) et Hostiles (Scott Cooper, E.-U., 2018).

7 Deadwood a reçu un excellent accueil public et critique, signe de l'efficacité d'une stratégie des chaînes câblées américaines visant à ériger la réinvention transgressive d'un genre en critère d'une nouvelle télévision de qualité, ainsi que le montre Achilleas Papakonstantis, « «How the West Was Won Again`. Les genres aux croisements du cinéma et des séries télé: l'exemple de Deadwood», Décadrages, $\mathrm{n}^{\circ}$ 32-33, 2016, pp. 73-90.

8 Westworld (Michael Crichton, E.-U., I973), qui a connu une suite (Futureworld, E.-U., I976) et s'est prolongé en une première série télévisée (Beyond Westworld, MGM Television/CBS, I980).

9 Pour un exemple science-fictionnel encore plus explicitement redevable du modèle vidéoludique de la représentation d'une immersion dans un environnement de western, voir le tome 3 de la série BD Les Aventures de Karen Springwell («Les joueurs de Convoi ${ }^{\mathrm{TM}}$ », I993), de Thierry Smolderen et Philippe Gauckler. 
s'empara déjà dès les années I970 avec les bornes d'arcade, s'est constitué en un véritable lieu de convergence médiatique grâce au jeu Red Dead Redemption (2010).

\section{Read Dead Redemption : un jeu de référence(s)}

Edité par Rockstar Games en 2010, Red Dead Redemption s'inscrit explicitement dans le genre westernien dont il reprend, condense et exhibe certains codes narratifs, esthétiques et «mondains». Le dessin qui figure sur la pochette du jeu représente le héros braquant son arme en direction de l'observateur (fig. I). Cette image, qui ne correspond à aucun moment du jeu dans la mesure où celui-ci repose sur une vue à la troisième personne, évoque l'un des «plans» d'un métrage des premiers temps, The Great Train Robbery (Edwin S. Porter, E.-U., I903), souvent considéré (à tort, dans la mesure où le terme de «western» n’existait pas

1/ Jaquette de la boîte du jeu Red Dead Redemption (2010) dans sa version pour

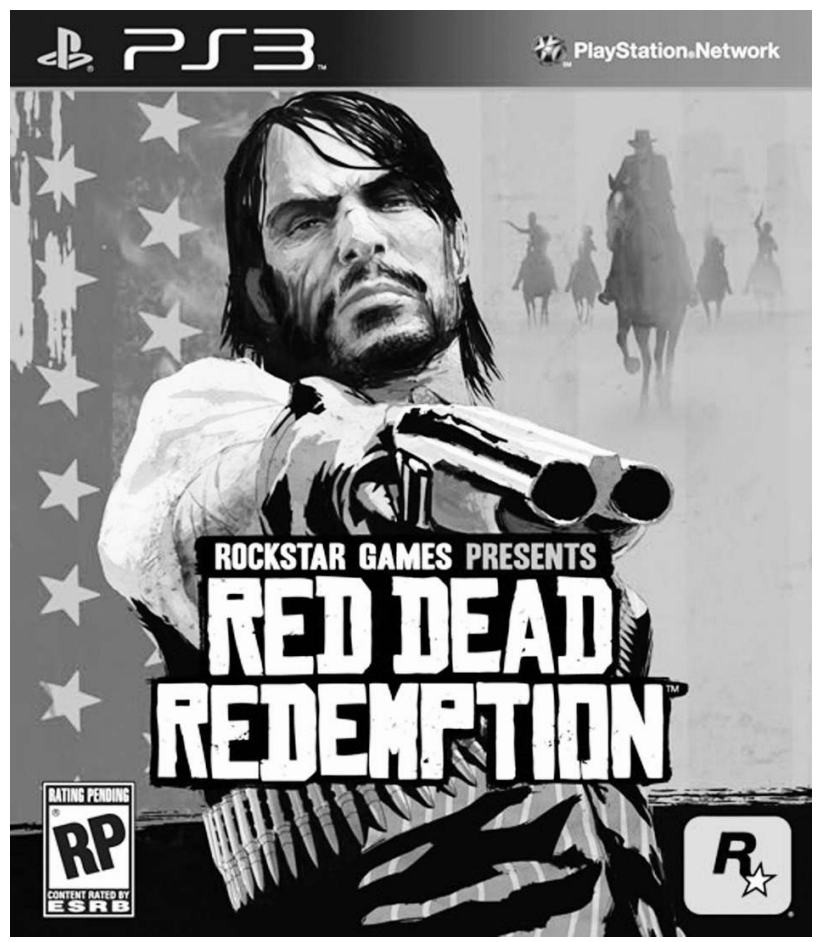




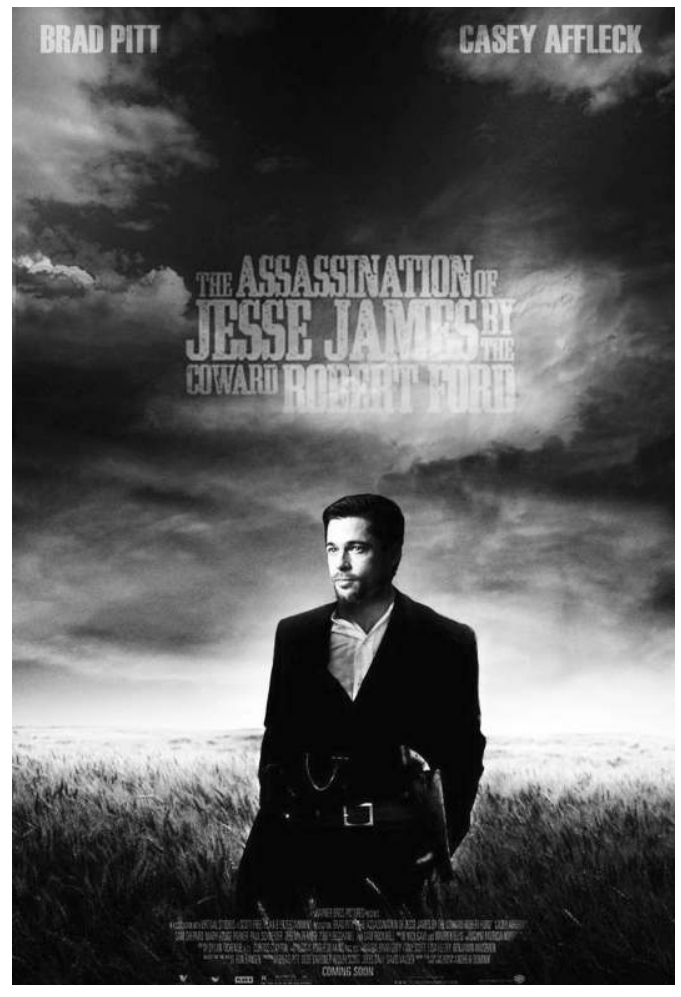

2/ Affiche promotionnelle pour le film The Assassination of Jessie James by the Coward Robert Ford (2007) mettant en valeur la star Brad Pitt dans le rôle du hors-la-loi charismatique, "centré» dans un environnement naturel rendu dans une image à la facture proche du cinéma de Terrence Malick, où les nuances de luminosité d'un ciel orageux renvoient à la «complexité» psychologique du protagoniste

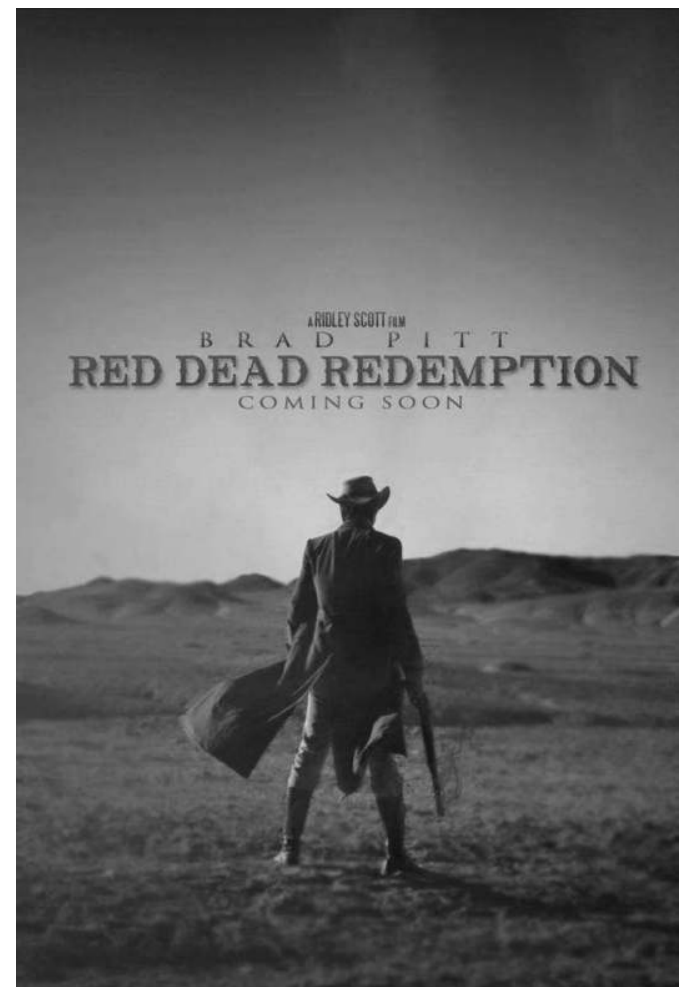

3/ La convergence médiatique comme partie intégrante de la culture fan actuelle: affiche fictive pour une adaptation cinématographique de Red Dead Redemption avec Brad Pitt encore) comme l'acte de naissance du genre cinématographique. La communauté des fans de Red Dead Redemption en a d'ailleurs espéré une adaptation au cinéma qui aurait compté dans sa distribution l'acteur Brad Pitt dans un rôle parent de celui tenu par la star dans The Assassination of Jessie James by the Coward Robert Ford (Andrew Dominik, E.-U./Canada/R.-U., 2007), un film qui suscita un regain d'intérêt pour le genre $\left(\right.$ fig. 2-3) ${ }^{10}$.
10 Voir par exemple le blog SlashFilm du 26 août 20 Io (www.slashfilm.com/ gigantic-rumor-brad-pitt-for-red-deadredemption) ou, dans le domaine du fandom, la création d'une affiche fictive ( fig.3) pour le film tiré du jeu: www.dorkly.com /post/44903/the-best-of-videogamemovie-posters. Consultés le 8 juillet 2018. 
11 Cette hybridation est également à l'œuvre dans plusieurs productions cinématographiques, des films de George A. Romero ou John Carpenter à Resident Evil: Extinction (2007) en passant par la série Walking Dead (AMC, dès 20Io) ou certains épisodes récents $(2014 ; 2017)$ de la franchise Planet of the Apes. Quelques films à petit budget destinés aux amateurs de «films de genre» inscrivent quant à eux au sein même du western des éléments gore (I Quattro de l'Apocalisse, Lucio Fulci, It., I975; The Burrowers, J.T. Petty, E.-U., 2008). La série de comic books de Jonathan Hickman et Nick Dragotta, East of West, débuté en 2013, présente une hybridité analogue, tout en fusionnant par ailleurs western et science-fiction à partir d'un postulat narratif basé sur une uchronie.

12 Les références internes à la diégèse de ces deux jeux portent d'ailleurs également sur la période de la naissance des studios dans les années igro. On peut à cet égard citer le décor de Intolerance: Love's Struggle Throughout the Ages (D.W. Griffith, E.-U., I9I6) dans L.A. Noire et, dans Red Dead Redemption, la mission secondaire intitulée «Lights, Camera, Action», où le joueur peut décider d'aider un personnage qui ambitionne de créer un studio de cinéma; Marston doit alors retrouver un acteur qui s'est reconverti en gunfighter et le dissuader de poursuivre dans cette voie, ce qui permet au jeu de thématiser la supériorité de la performance de l'avatar sur celle d'un «tireur de cinéma».
Red Dead Redemption se présente comme un jeu d'action-aventure se jouant sur consoles PlayStation 3 ou Xbox 360 , et proposant une figuration de l'espace en $3 \mathrm{D}$ polygonale. Il a rencontré un énorme succès - sa suite, annoncée pour octobre 2018 , est très attendue -, au point de représenter peut-être à ce jour la production westernienne du $\mathrm{XXI}^{\mathrm{e}}$ siècle la plus connue du grand public, et la plus unanimement valorisée dans l'espace de réception. Nous l'examinerons ici strictement dans son mode «histoire» (joueur unique contre la machine), en faisant l'impasse sur le mode «multijoueur» et sur l'extension intitulée Undead Nightmare, qui, hybridant le western et l'horreur, invite l'utilisateur à revisiter, grâce à un procédé métaleptique - un récit enchâssé lu à un enfant y «contamine» le récit-cadre -, les mêmes lieux désormais désertés et hostiles, la population s'étant transformée en zombies ${ }^{11}$.

Rockstar Games adopte avec Red Dead Redemption une stratégie visant à inscrire un monde vidéoludique dans une référence à un genre cinématographique. Cette démarche sera reconduite l'année suivante avec la sortie de L.A. Noire (20II), qui renvoie dès son titre au genre du film noir et plus spécifiquement à des enquêtes à la James Ellroy menées par des détectives dans l'envers obscur du décor clinquant d'Hollywood. Dans les deux cas, une représentation audiovisuelle créée par des développeurs et générée par une machine informatique capitalise sur l'attrait cinéphilique des consommateurs à l'égard de la période classique d'Hollywood ${ }^{12}$, tout en éveillant l'intérêt des gamers d'aujourd'hui pour cette production des années I940-1950. Toutefois, l’inévitable réflexivité induite par une transposition médiatique si tardive s'accompagne de l'intégration de pastiches ultérieurs qui ont contribué à déplacer certaines normes: L.A. Noire réinterprète un genre de l'aprèsguerre à travers la loupe déformante du «néo-noir», tandis que Red Dead Redemption représente l'Ouest américain du début du $\mathrm{xx}^{\mathrm{e}}$ siècle à l'aune de l'imagerie exubérante du «western spaghetti». Outre les emprunts évidents aux films des trois «Sergio» (Leone, Corbucci et Sollima) au niveau du bruitage, des situations narratives, des décors (en particulier dans les espaces «mexicains» du jeu) et de la représentation d'actions violentes, Red Dead Redemption imite en effet ostensiblement, et cela dès que résonne un harmonica accompagné d'une mélodie sifflotée sur l'écran du menu, les thèmes musicaux composés par Ennio Morricone pour la «trilogie du dollar» de Sergio Leone. Clint 
Eastwood y incarnait un personnage sorti de nulle part, habituellement désigné dans les discours parafilmiques par la formule énigmatique de «l'homme sans nom ${ }^{13}$. Cette sorte de «présence-absence» dépourvue de toute consistance psychologique (et a priori de sens moral) ${ }^{14} \mathrm{se}$ prête idéalement à une transposition en un avatar vidéoludique dont les propriétés sont, mutatis mutandis, similaires à celle du protagoniste filmique, dans la mesure où tous deux se définissent à travers leurs seules actions.

\section{Le " cowboy " de pixels, un héros doublement anachronique}

Le héros de Red Dead Redemption, quant à lui, a un nom: John Marston. En écho à la notion de «rédemption» présente dans le titre pour des raisons qui excèdent semble-t-il le seul intérêt phonétique de l'allitération, le personnage du jeu est muni d'un arrière-plan psychologique défini (en l'occurrence par la culpabilité et la nécessité de se racheter) - plutôt selon le schéma narratif «christique» qui caractérise les westerns réalisés par Corbucci puis par Eastwood. En effet, dans la version «histoire» du jeu, John Marston est un ancien hors-la-loi contraint par les agents fédéraux qui ont pris son épouse et son fils en otage à poursuivre et à éliminer ses anciens comparses (ou «camarades de jeu») au nom de l'édification de la civilisation. A l'instar de Pat Garrett dans le film de Sam Peckinpah (Pat Garrett and Billy the Kid, E.-U., I973), dont l'intrigue semble avoir été l'une des sources d'inspiration pour les scénaristes de la production Rockstar, Marston est une relique d'un monde «sauvage» en voie d'extinction agissant au nom de valeurs qui supposent sa propre perte.

D'ailleurs, comme dans The Ballad of Cable Hogue (Sam Peckinpah, E.-U., I970), la diégèse du jeu prend place après la conquête de l'Ouest (le récit est situé entre I9II et I9I4), soit à une époque où cette dernière fait déjà figure de mythe. Les signes de la «modernité», évidents dans la ville de Blackwater, sont présents dès le tout début du jeu. En effet, dans la cinématique inaugurale ${ }^{15}$, alors que John Marston débarque d'un bateau à roues à aubes dans un port marchand où l'attendent deux agents qui l'escortent jusqu'à la gare ferroviaire, un insert permet au «spectateur» - car l'utilisateur n’a pas encore véritablement «joué», si ce n’est en lançant le jeu - de se focaliser sur un élément d'abord montré de manière subreptice à l'arrière-plan: il s'agit d'une grue pivotant pour débarquer
13 Dans la version originale italienne, le héros se prénomme Joe, mais la version anglaise optait pour l'absence de dénomination du protagoniste; de même, pour la version anglophone de Per qualche dollaro in più (It./Esp./R.F.A., I965), «le Manchot» fut rebaptisé «l'homme sans nom» (voir Christopher Frayling, Il était une fois en Italie. Les westerns de Sergio Leone, Paris, La Martinière, 2005, p. 43 et p. 47). A propos de la version française du premier opus, Pour une poignée de dollars, voir notre commentaire dans « Le doublage au sens large: de l'usage des voix déliées », Décadrages, $\mathrm{n}^{\circ} 23-24,2013$, pp. 53-55.

14 Au cinéma, le héros témoigne toutefois en général (et en dépit des apparences) d'un code moral propre, inspiré des films de samouraïs ou de gangsters. Dans Red Dead Redemption, le réglage idéologique motive l'une des nombreuses limites auxquelles est assignée la manipulation de l'avatar. Ainsi, par exemple, le joueur peut difficilement se comporter en hors-la-loi sanguinaire, car si la cote d'honneur qui constitue l'une des jauges de l'interface dégringole, l'avatar a tôt fait d'être encerclé par les forces de l'ordre et abattu; de même, s'il est harangué par une prostituée, l'avatar répond mécaniquement, sans permettre au joueur d'interagir, qu'il n'est pas intéressé par la proposition.

15 Concernant la notion de «cinématique», voir l'article de Selim Krichane et Yannick Rochat dans le présent dossier. 
Images extraites de la cinématique inaugurale de Red Dead Redemption, dans laquelle apparaissent les icônes de l'ère industrielle et d'une «vision mobile» symptomatique de

la modernité: le train et l'automobile

16 Notons que John Marston est amené lors d'une mission secondaire ( Deadalus and Son») à aider un inventeur à réparer son planeur. Comme le souligne le jeu de mot du titre («Deadalus»), la tentative de vol sera fatale à l'infortuné pionnier. Les composantes narratives du jeu fonctionnent donc à la défaveur de l'implantation de la technologie, comme s'il sagissait de maintenir encore la temporalité du mythe. Ainsi, dans un autre passage du jeu, on apprend que la ligne téléphonique nouvellement installée dans le bureau du shérif et qui fait la fierté de ce dernier ne fonctionne pas, ce qui motivera le déplacement à cheval de l'avatar.

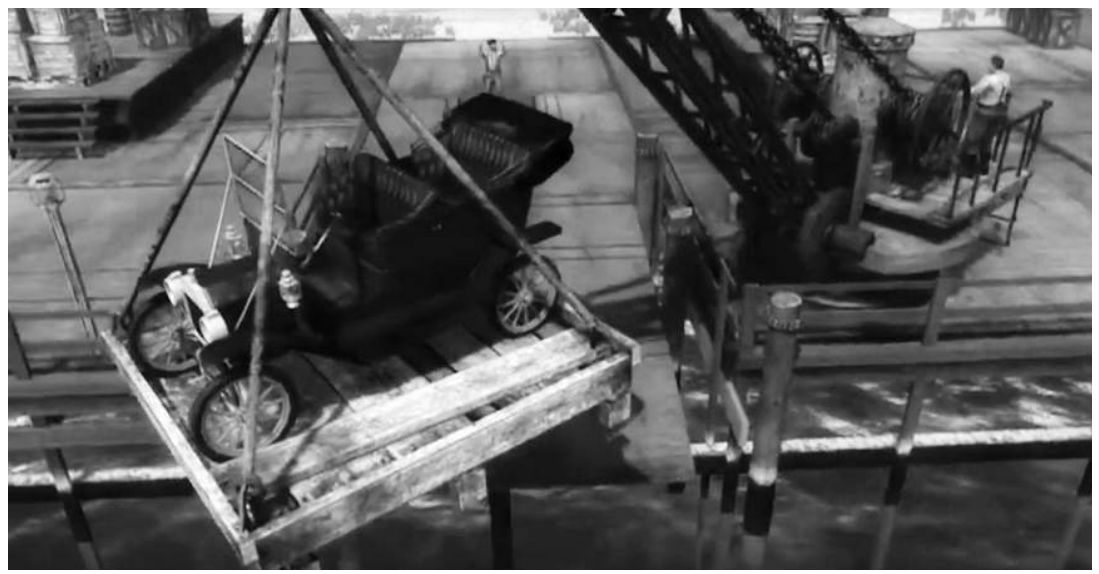

4

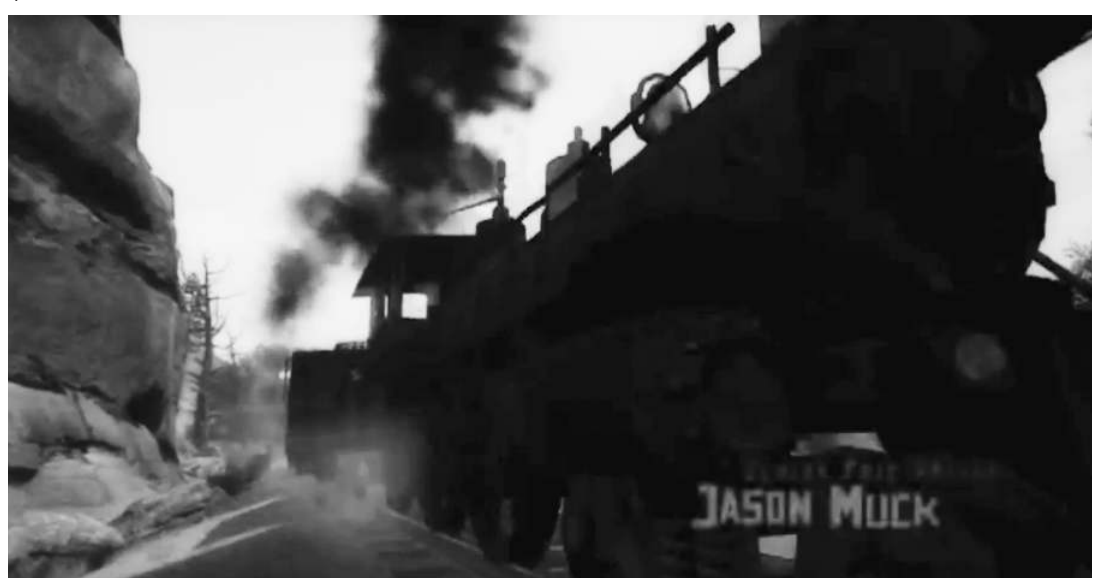

5

d'un navire une Ford T (fig. 4), automobile qui représente le produit paradigmatique du taylorisme. Le recours à un montage apparent dans un régime de visualité où prime l'effet de continuité renforce le contraste entre la dégaine typique du «cowboy» et le siècle de la machine. Durant le générique qui reproduit la pratique cinématographique d'une succession de mentions écrites sur fond noir, nous voyons John se rendre dans la ville (fictive) d'Armadillo en prenant le train, autre moyen de locomotion emblématique de l'ère industrielle ${ }^{16}$ (fig. 5$)$. On peut dire que cette 
ouverture suggère un rapport de simultanéité entre l'arrivée du héros et celle de la modernité, le premier ayant pour mission d'asseoir la seconde encore fragile, fût-ce à son corps défendant. Au vu de cette thématisation de la technique (au sein d'une représentation convoyée par un médium hautement technologique), on ne s'étonnera pas que Red Dead Redemption offre au joueur la possibilité d'assister à une séance de cinéma.

John Marston, personnage d'un autre temps, est, on l'a dit, voué à disparaître après avoir servi une cause qu'il méprise: une fois sa tâche accomplie - en l'occurrence une série de «missions» proposées par le jeu comme autant de boucles actionnelles multipliées à l'envi au sein d'un arc narratif plus vaste -, les représentants de l'Etat fédéral se retourneront contre lui. Le joueur se trouve alors dans une situation d'impuissance a priori incompatible avec l'immersion vidéoludique ainsi qu'avec la gratification de l'habilité qui est en général offerte ad libitum par les jeux vidéo de type action-aventure, où le "game over» ne constitue qu'un état de pause provisoire, à l'instar des «morts» que le personnage de Marston a pu subir jusque-là à l'occasion d'interruptions marquées par une ponctuation musicale et une mention lapidaire («Mort»), avant que John ne «renaisse» à l'emplacement de la dernière sauvegarde (ou à l'entame de l'action en cours). C’est la distance imposée

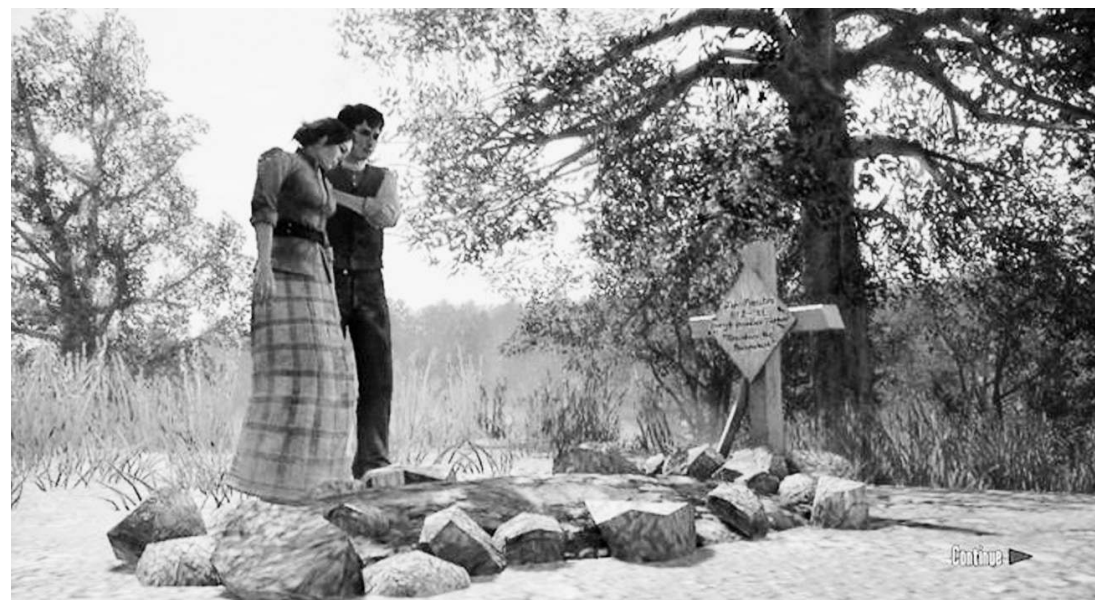

6/ La mort du père, ou le joueur rattrapé par le fatum du récit: d'un avatar à l'autre s'opère inéluctablement un transfert qui obéit aux normes patriarcales du western 
17 «La contrepartie [du] plaisir confortable de l'immersion réside dans la prise de conscience diffuse d'une certaine impuissance du spectateur dans son rapport au monde. [...] [O]n peut remarquer une tendance régulière à l'expression d'une inquiétude concernant la difficulté pour les personnages qu'ils [jeux vidéo ou films récents] mettent en scène à avoir prise sur le monde. [...] [R]elevons par exemple cette mise en scène du choix dans des jeux tels que Heavy Rain (Quantic Dream, 20ıо), The Walking Dead (Telltale Games, 20I2) ou les jeux à monde ouvert développés par la société Rockstar (GTA IV et $V$, L.A. Noire, Red Dead Redemption,...)» (Raphaël Koster, «En quête de réalités : l'expérience immersive comme mode d'engagement du spectateur», dans Alexis Blanchet (éd.), Jeux vidéo/cinéma. Perspectives théoriques, Paris, Editions Questions théoriques, 20I7, p. III).

18 L'image du héros faisant face à une pierre tombale qui emblématise un revers du destin est récurrente dans les films de John Ford par exemple (Young Mr.Lincoln, E.-U., I939; My Darling Clementine, E.-U., I946; She Wore a Yellow Ribbon, E.-U., I949).

19 En effet, Jack ne se lance dans l'aventure qu'après le décès de sa mère. La tombe ponctue ainsi le récit et précise son ancrage historique, puisque l'épitaphe de la pierre tombale comporte l'indication «I873-I9II".

20 Jesper Juul, The Art of Failure: An Essay on the Pain of Playing Video Games, Cambridge etc., MIT Press, 2013. Notons que Juul mentionne l'exemple de Red Dead Redemption, qu'il conçoit comme «une réponse au problème logique résultant de la combinaison du jeu avec la narration» (p. I05).

au joueur par le finale de la première partie qui fait tout le sel, sinon la «cinématographicité», de Red Dead Redemption, à l'instar d'autres jeux qui court-circuitent ainsi momentanément le potentiel d'action sur le monde que promet un médium défini par son interactivité ${ }^{17}$. Ce renversement survient lorsqu'une escouade d'agents dissimulés dans la grange attenante à la ferme de Marston le surprend, ouvrant le feu lors d'un imparable assaut qui rappelle la séquence finale de Butch Cassidy and the Sundance Kid (E.-U., I969). Mais alors que le film de George Roy Hill s'achevait sur une fin ouverte en l'espèce d'un arrêt sur image dans l'ultime plan, John Marston, une fois garantie la fuite des siens, périt sous les balles. Une cinématique s'enclenche alors qui cantonne le joueur au rôle de simple spectateur d'un pivot narratif culminant dans l'image de la mère et du fils recueillis devant la tombe du père (fig. 6$)^{18}$.

Au moment du décès de John, son patronyme fait office de relais, puisque le nouvel avatar que le joueur est amené à actionner n'est autre que son fils, John «Jack» Marston Jr., pour la survie duquel John s'est sacrifié et qui n'aura de cesse, si l'usager «joue le jeu», de venger son père dans un monde en mutation - après une ellipse ${ }^{19}$, les villes traversées auparavant se seront désormais considérablement urbanisées, la segmentation du jeu en deux parties identifiées comme telles par des mentions écrites (même si la seconde est considérablement plus brève en termes de progression) renforçant la linéarité du récit et conférant au joueur le sentiment d'une historicité du monde représenté. Partant d'une discussion de l'ouvrage The Art of Failure de Jesper Juul ${ }^{20}$, qui souligne combien les jeux vidéo reposent sur une expérience de l'échec qui, paradoxalement, n’enraie pas le plaisir ludique, le théoricien québécois Simon Dior observe que la mort «définitive» du héros de Red Dead Redemption, irrémédiablement singulative dans un médium dont la représentation se caractérise par la répétition, «va a contrario de la jouabilité elle-même» en imposant l’inévitabilité de la tragédie: «L'échec, ici, n'est pas réel (car le joueur a bel et bien terminé le jeu), mais uniquement fictionnel $»^{21}$.

21 Simon Dior, «Red Dead Redemption, l'échec fictionnel et la tragédie», en ligne: www.simondor.com/blog/2014/o4/reddead-redemption-echec-fictionnel.html. 
La dissociation entre la temporalité de la diégèse vidéoludique et l'expérience du joueur vécue dans l'immédiateté de l'interaction se creuse dans l'ellipse, et s'affiche via le passage d'une focalisation interne sur John à un nouvel ancrage sur Jack. Ce changement de repère occasionne une forme d'équivalence vidéoludique de la temporalité filmique, ainsi qu'une réappropriation d'un topos éculé de la critique qui consiste à qualifier certains westerns de «crépusculaires»-dans ces films tardifs (à partir des années I960) 22, on y lit aussi le crépuscule du genre luimême -, le sombre défaitisme y signant la fin d'une ère. Pour reprendre une terminologie appliquée à la technologie informatique qui précisément ici s'efface devant un monde du passé dans lequel le personnage luimême devient anachronique, on pourrait dire que le récit fait peser sur le héros la menace d'une «obsolescence programmée». Le paradoxe d'une représentation numérique d'une époque antérieure à l'informatique est en quelque sorte thématisé dans Red Dead Redemption à travers un motif caractéristique du genre.

\section{$R D R$ : au début était le revolver}

Read Dead Redemption s'inscrit dans le prolongement d'une précédente production de Rockstar Games destinée à la console PlayStation 2, Red Dead Revolver, sortie en 2004 et qui a fait l'objet dix ans plus tard, sans doute en raison du succès considérable du produit parent qui lui a succédé, d'une ressortie sur $\mathrm{PS}_{4}$ dans une version remasterisée. L'horizon référentiel de l'univers du jeu était déjà le «western spaghetti», ainsi que le signifie très explicitement la reprise de compositions musicales réalisées pour des films italiens, telles que le thème principal de Lo Chiamavano King (Giancarlo Romitelli, It., I97I), ou des images en format large montrant l'arrivée du héros dans un village, à l'instar de celle de l'énigmatique solitaire incarné par Eastwood dans Pale Rider (Clint Eastwood, E.-U., I985), et surtout dans le film qui est à l'origine d'un nouveau type de personnage, Per un pugno di dollari (Sergio Leone, It./Esp./R.F.A., I964). D’ailleurs, le poncho mexicain devenu célèbre du personnage interprété par Eastwood dans le premier western de Leone (fig.7) peut être acheté en tant qu'item dans Red Dead Revolver (avec une fenêtre d'opportunité limitée au cours de la mission intitulée «Chasseur de primes»), et constituera de manière plus marquante l'une des tenues à choix proposées
22 Aussi le dernier chapitre de l'ouvrage de Georges-Albert Astre et Albert-Patrick Hoarau, Univers du western (Paris, Seghers, 1973, pp.332-390), est-il intitulé «westerns crépusculaires». 
7/ Affiche promotionnelle pour la sortie de la version anglophone de Per Qualche dollaro in più (1965)

8/ Quand John Marston se prend pour Clint Eastwood: les attributs vestimentaires de l'avatar
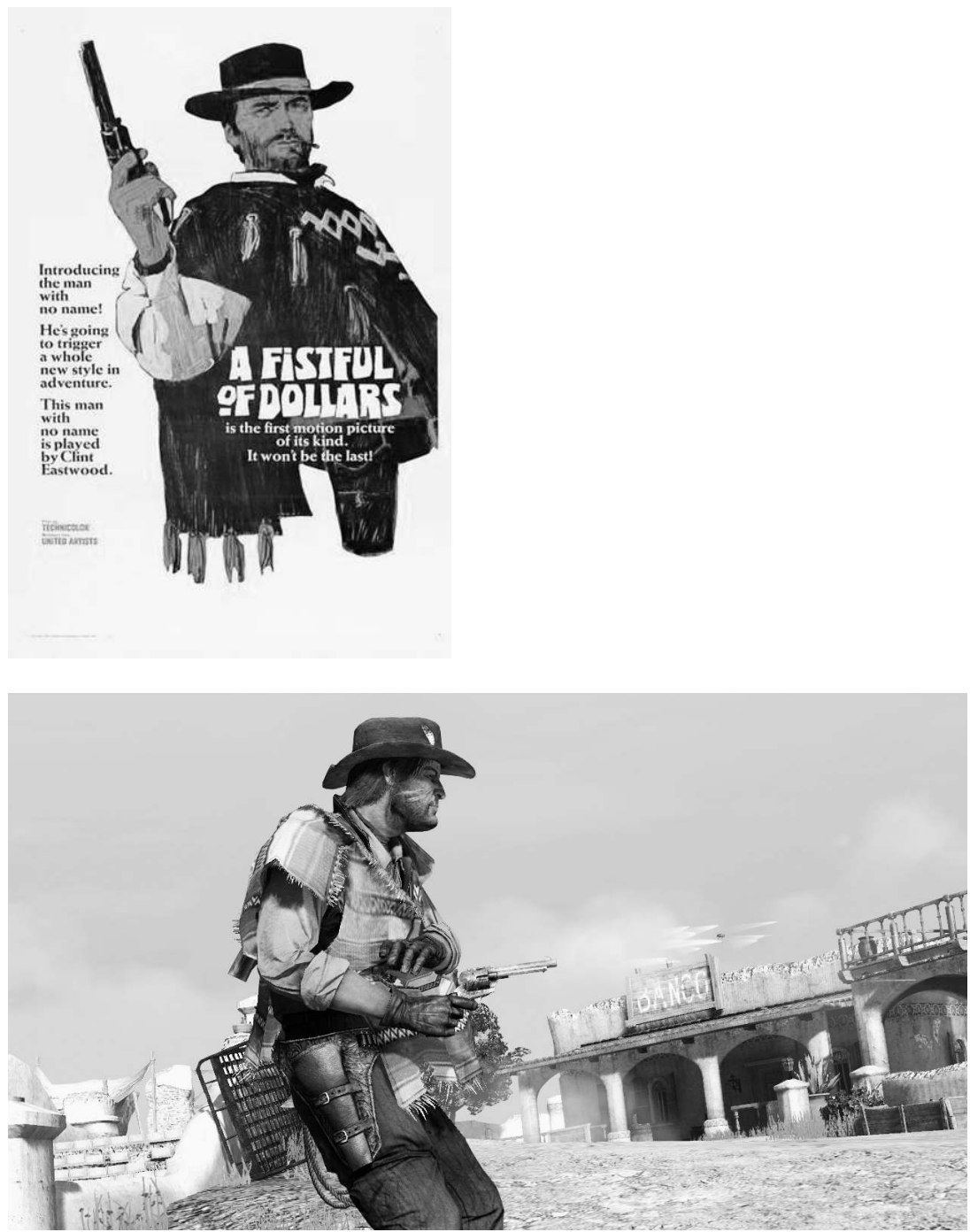

dans Red Dead Redemption, où tant John que Jack peuvent la revêtir (fig. 8).

Le culte cinéphilique pour certains détails «profilmiques» trouve dans une telle transposition vidéoludique une satisfaction particulière 
qui tient à l'importance conférée dans le jeu à des objets dont le joueur peut faire l'acquisition pour en pourvoir son avatar, selon un processus basé sur l'accomplissement de missions successives corrélé à l'acquisition progressive de nouvelles ressources. Si le poncho participe à la construction de la désinvolture du personnage, il n'a pas d'incidences sur l'action, contrairement à l'arsenal que porte sur lui John Marston, et dont la composition obéit à une logique évolutive qui est celle de la progression dans le jeu. La «trilogie du dollar» de Leone témoigne d'une passion évidente pour les armes à feu et le «professionnalisme» avec lequel en usent les chasseurs de primes, capables de tirs chirurgicaux. Dans la première séquence de Per qualche dollaro in più (It./Esp./R.F.A., I965), le personnage interprété par Lee van Cleef choisit soigneusement parmi ses «instruments de
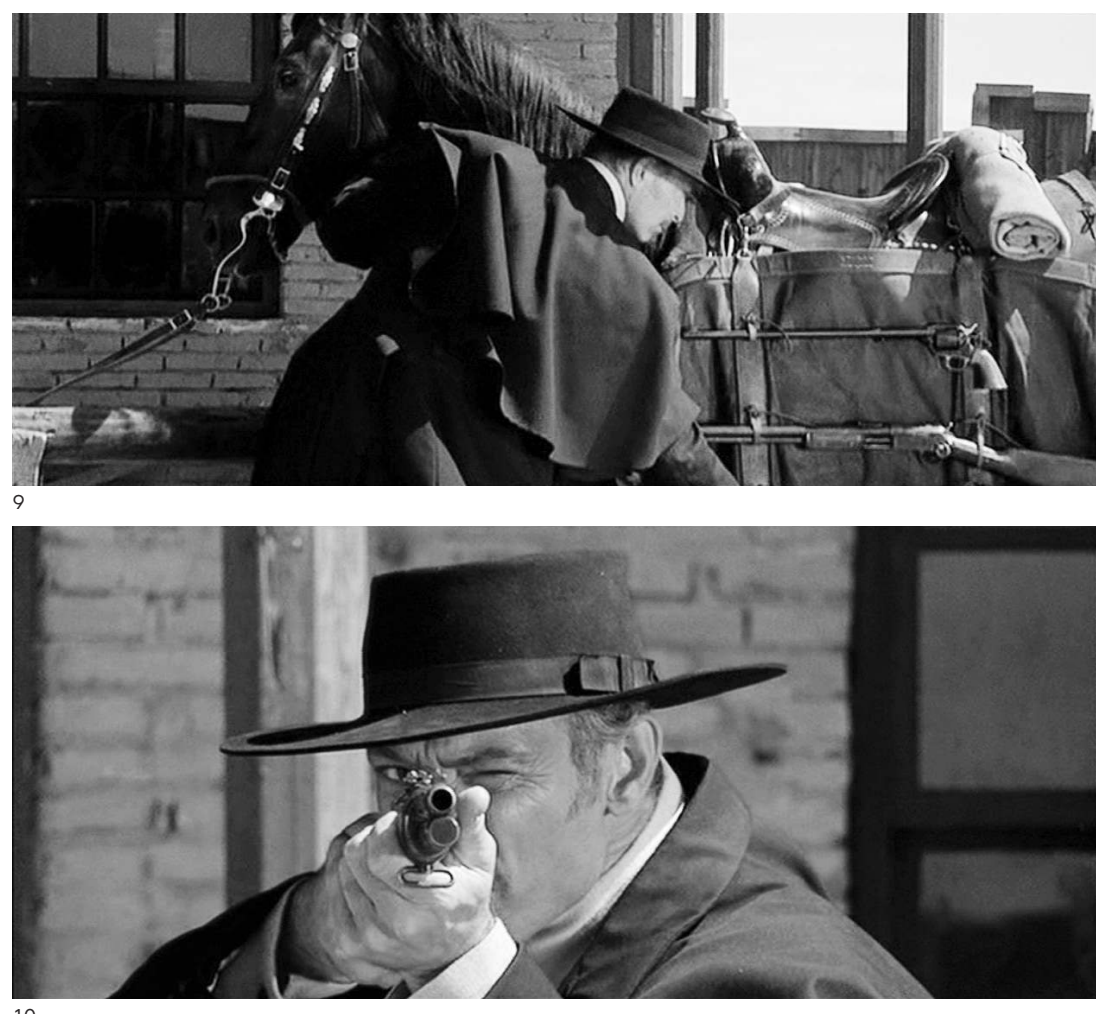

L'arsenal du colonel Mortimer dans Per Qualche dollaro in più (1965) 


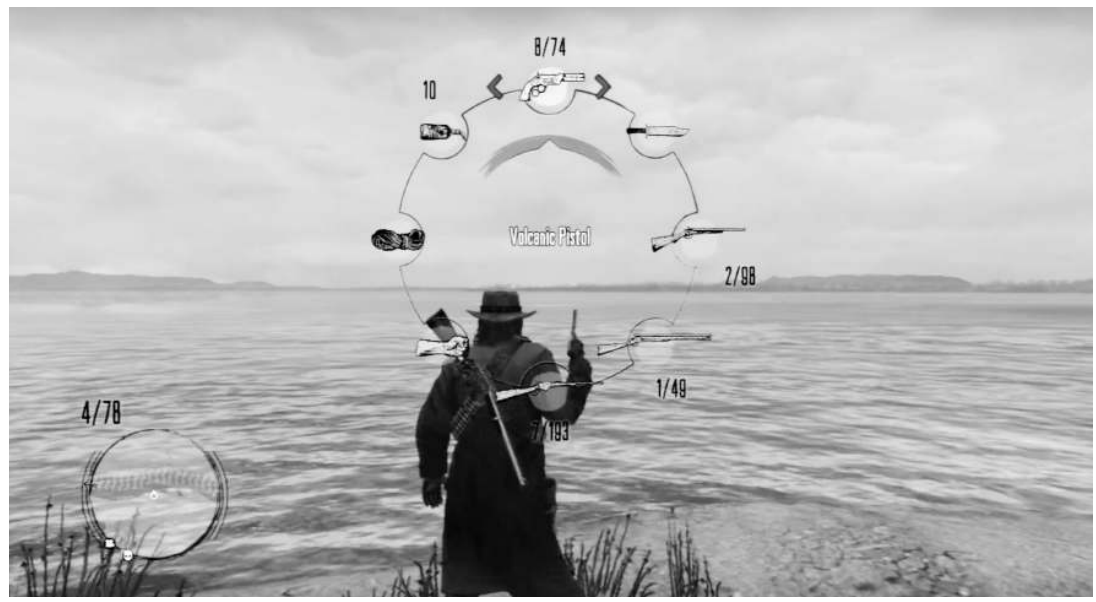

11

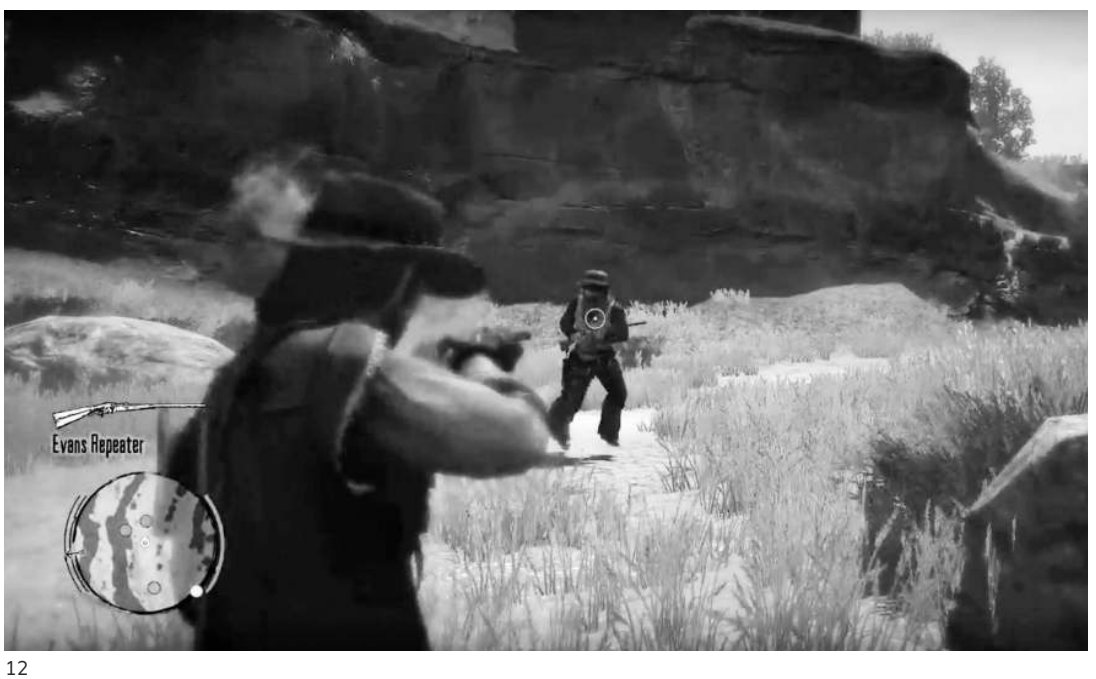

travail» disposés dans la sacoche fixée à la selle de son cheval le fusil qui lui permet d'abattre sa proie en fuite à l'autre extrémité de la rue centrale ( fig. 9-Io). Dans Red Dead Redemption, le joueur peut aussi, à tout moment, faire apparaître les armes qui sont à sa disposition et opter pour celle qui convient le mieux à la situation en fonction de caractéristiques techniques spécifiées à l'écran (fig. II-I2). L'équipement se renforce au cours 
du jeu: l'avatar peut en effet faire l'acquisition d'armes en magasin, le joueur utilisant ainsi l'argent des primes reçues pour avoir notamment capturé ou tué des hors-la-loi, dans des séquences qui ressemblent à des campagnes promotionnelles pour la NRA (fig. I3-I4).

Dans une perspective d'analyse des contenus politiques, Olivier Mauco, qui définit le game design comme «l'art d'écrire des dispositifs
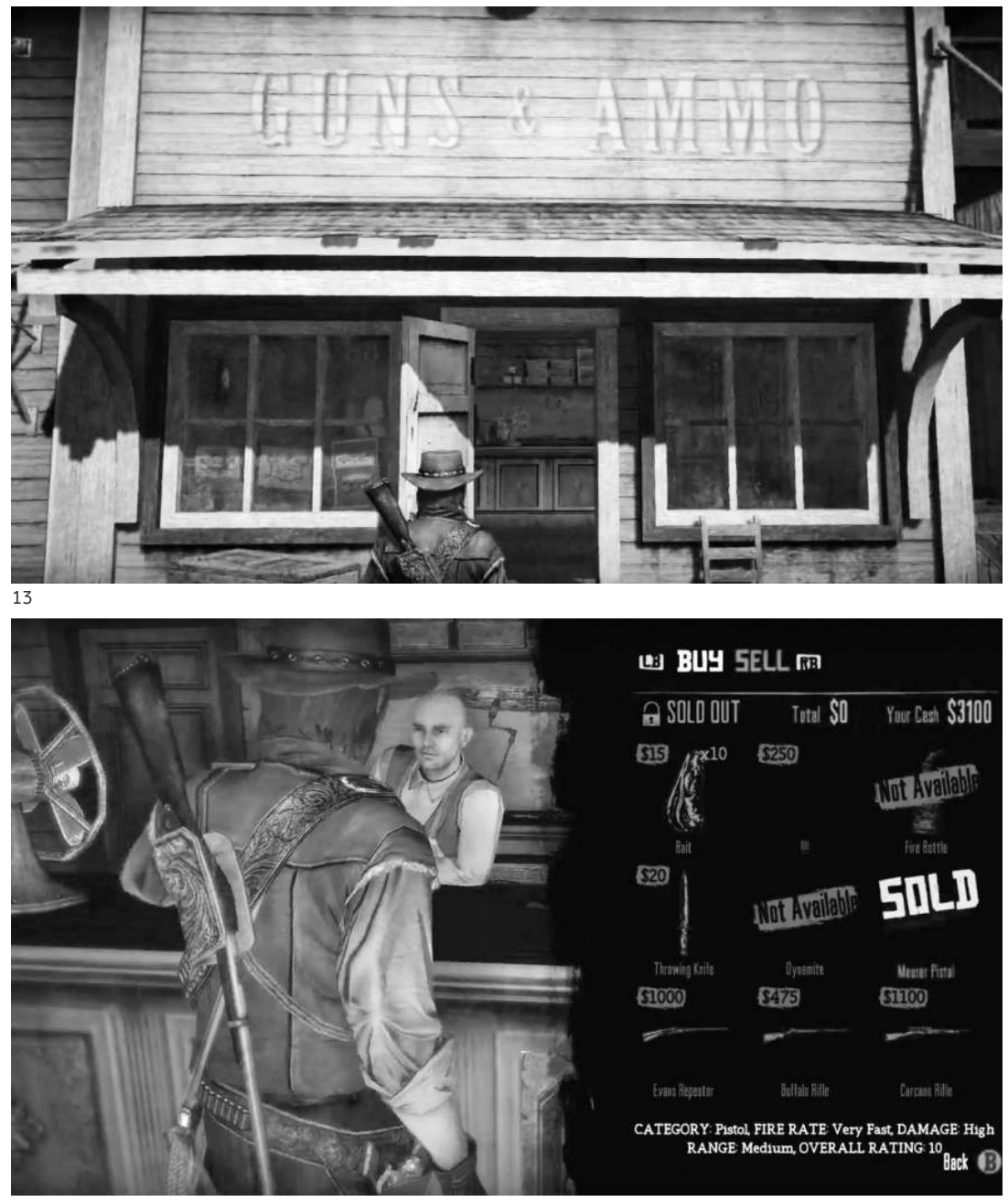

Les emplettes du "cowboy " dans Red Dead Redemption 
La cible dans la mire de l'avatar: Red Dead Revolver / Redemption

23 Olivier Mauco, GTA IV. L'Envers du rêve américain. Jeux vidéo et critique sociale, Paris, Questions théoriques, 2013, p. 53 .
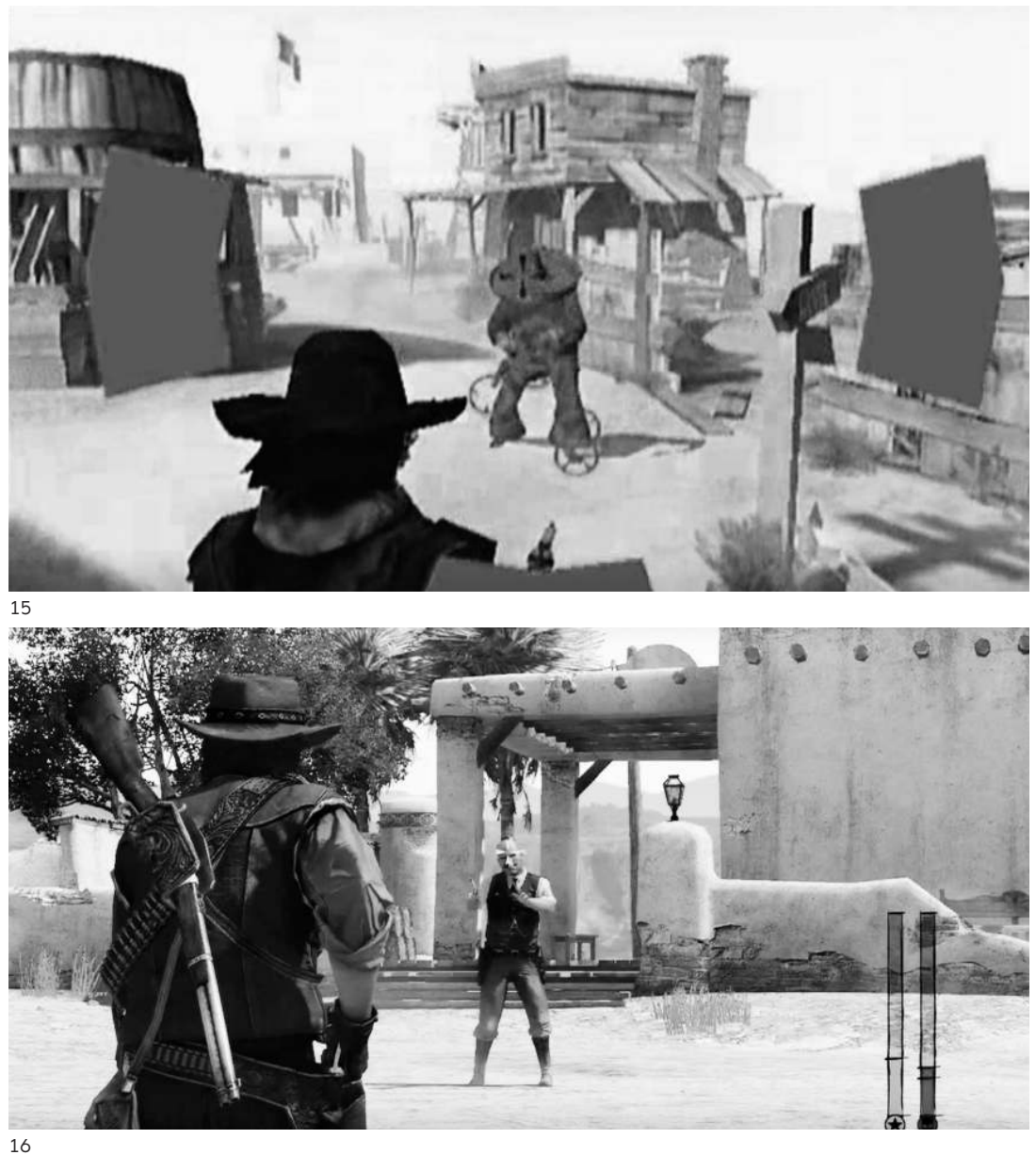

de pouvoir par la modélisation de systèmes (le game) et de rapport aux systèmes (le play rationalisé en gameplay)», fait observer que «le jeu vidéo met en scène le pouvoir dans la capacité de l'avatar à évoluer dans le monde ludo-fictionnel.» ${ }^{23}$ Or dans les deux Red Dead, cette capacité de l'avatar à s'imposer dans son environnement est alimentée par une mécanique de jeu permettant de tirer plusieurs salves successives en direction de points d'impact présélectionnés grâce à des mires qui apparaissent à l'écran pendant que les mouvements de la cible sont figés 
ou ralentis (fig. I5-IO). Durant cette phase de courte durée, le temps est suspendu, sauf pour l'avatar-tireur, dont la rapidité des réflexes, représentée comme au cinéma par un ralenti paradoxal (qui exprime l'extrême rapidité par la lenteur), équivaut à celle du «cowboy» légendaire qui, comme l'écrivait parodiquement Morris à propos de Lucky Luke, «tire plus vite que son ombre». Cette possibilité offerte par le gameplay, nommée «Dead Eye Targeting», est emblématique d'une (con)fusion presque complète dans les Red Dead entre regarder et tirer, le médium vidéoludique reconduisant le constat de Paul Virilio à propos de l'entrelacement des généalogies respectives de l'armement et des appareils de prise de vues, dans la mesure où, «pour l'homme de guerre, la fonction de l'arme c'est la fonction de l'œil» ${ }^{24}$. Comme l'illustrent «d'entrée de jeu» le tout premier plan de The Last Wagon (Delmer Daves, E.-U., I956) ou l'image de pré-générique dans Per qualche dollari in più (I965), le paysage se donne le plus souvent à voir dans les westerns comme le lieu d'apparition d'une cible potentielle ${ }^{25}$. L'usage de fusils à lunette dans Red Dead Redemption exacerbe l'alignement du regard et de la cible (fig. I7) qui, au cinéma, s'accompagne en général d’un décentrement ${ }^{26}$ permettant de conserver l'acteur dans le champ (fût-ce dans une image qui ne le montre qu'en amorce, comme dans Winchester'73, Anthony

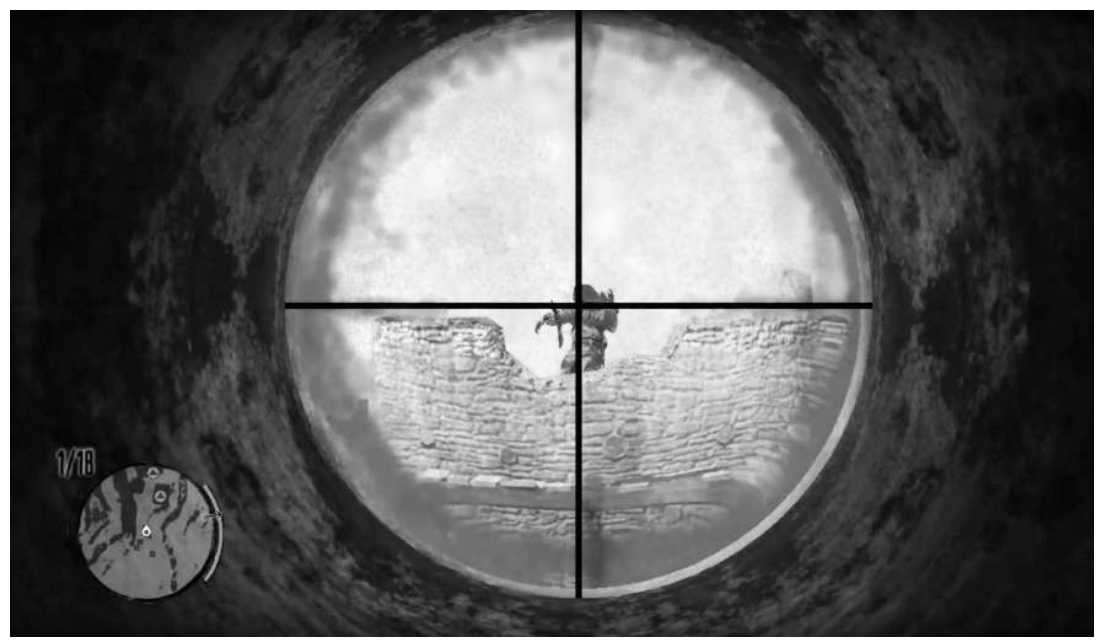

24 Paul Virilio, Guerre et cinéma I. Logistique de la perception, Paris, Cahiers du cinéma/Editions de l’Etoile, i984, p. 26.

25 Dans ces deux incipit filmiques qui montrent un cavalier au loin se déplacer calmement dans un vaste environnement naturel puis soudain se faire abattre, le spectateur est d'abord amené à penser qu'il s'agit simplement de poser le décor, puis comprend que le protagoniste anonyme qui y déambule est pris pour cible.

26 En effet, les vues subjectives ne sont que très ponctuelles dans les westerns, le spectateur étant plutôt invité à regarder un personnage dégainer et tirer (surtout si ce dernier constitue le pôle identificatoire du film). On trouve par exemple des plans en caméra subjective (associés au regard d'un protagoniste secondaire) dans Hombre (Martin Ritt, E.-U., I967, à rh45'r9" et rh47'24"), Gli Specialisti (Sergio Corbucci, It./F./R.F.A., I969, à Ih25'O4") ou The Quick and the Dead (Mort ou vif, Sam Raimi, E.-U., à I's5").

17/ Voir I'histoire américaine par le petit bout de la lorgnette (Red Dead Redemption) 
18/ Le regard ancré dans un tireur en amorce: Winchester'73 (1950)

27 Nous renvoyons sur ce point à notre article «L'inquiétante étrangeté du found footage horrifique», Décadrages, $\mathrm{n}^{\circ}{ }_{2} \mathrm{I}^{-}$ 22, 2012, pp. 146-ı65. En ligne: https:// journals.openedition.org/decadrages/ 687.

28 A propos des implications immersives de ce procédé popularisé par le jeu Gears of War (Epic Games, Microsoft Game Studios, dès 2006) qui consiste à maculer la surface de «l'objectif» (virtuel), voir Selim Krichane, «La Caméra imaginaire. Histoire et théorie des modes de visualisation vidéoludique», thèse de doctorat soutenue à l'Université de Lausanne en mai 2017, pp.389-390, à paraître chez Georg sous le titre La Caméra imaginaire. Jeux vidéo et modes de visualisation.

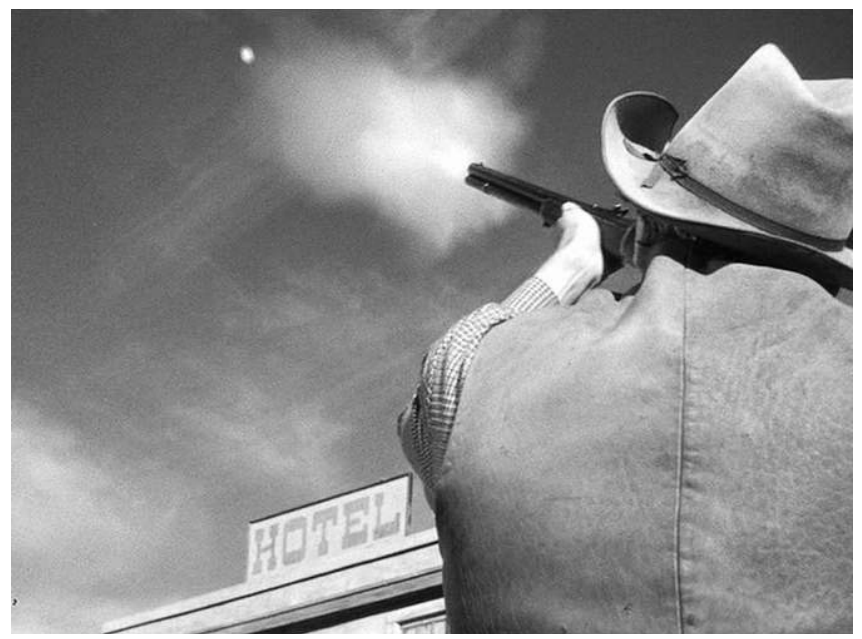

Mann, E.-U., I950, où le personnage interprété par James Stewart est visualisé sur un mode parent des avatars de jeux à la troisième personne (fig. I8).

Le deuxième Red Dead offre par ailleurs une surenchère en termes d'hémoglobine (la promesse du «rouge» figurant dans le titre est tenue), des éclaboussures venant parfois se projeter sur «l'objectif» (il en va de même des gouttes de pluie, comme dans le jeu Heavy Rain, 20ıо), comme si l'image résultait d'une captation par une caméra située au cœur de l'action. On retrouve là un «effet documentarisant» dont usent également les fictions cinématographiques, notamment horrifiques ${ }^{27}$, et qui peut paraître plus absurde encore dans une représentation entièrement générée en temps réel par une machine si le jeu n'obéissait pas, via l’imitation de séquences de films, à une esthétique "photoréaliste» au service d'une visée immersive ${ }^{28}$. De tels modes de représentation soulignent combien l'accès à la représentation vidéoludique est «filtrée» par des pratiques dominantes qui se sont imposées dans d'autres médias. En effet, ainsi que le montre Carl Therrien dans le cadre d'une étude historiographique de l'usage du label «First Person Shooter» (dont il souligne l'association récurrente, dès le début des années I990, avec l'exploration libre d'un espace $3 \mathrm{D}$ ) dans les textes promotionnels et journalistiques, ce 
filtre s'observe également au niveau des discours qui conçoivent la "première personne» dans le cadre de la seule configuration visuelle (plutôt que dans un lien plus large à l'expérience vidéoludique), les jeux vidéo demeurant «appréhendés et formatés par le langage selon une conception picturale/cinématographique ${ }^{29}$. Il en va de même, dans Red Dead Redemption, des variations de vitesse (on pense aux ralentis dans les films de Sam Peckinpah), des écrans couverts de rouge, des zooms permettant de mieux identifier la zone à viser ou des fondus au noir. Lors de fusillades s'opère par conséquent un décollement de la temporalité diégétique par rapport à celle de l'expérience du joueur que l'on peut considérer comme similaire à celui postulé par l'accès, en tout instant du jeu, à diverses fonctionnalités (cartes, armes, argent, habillement, etc.), à la différence près que nous ne quittons pas l'espace diégétique dans le cas de ce temps suspendu conjugué à la possibilité d’une variation de l'angle de «prise de vue» et d'un déplacement du tireur. La coprésence de deux vitesses actionnelles et cette forme de panoptisme jubilatoire définissent un mode de représentation communément qualifié de «Bullet Time» depuis son utilisation remarquée dans le film Matrix (frères Wachowski, E.-U., I999). Désormais commune au cinéma et aux jeux vidéo, l’imagerie numérique favorise de tels transferts esthétiques d'un médium à l'autre ${ }^{30}$ : le récit de Matrix justifiait le retrait du tireur hors l'espace traversé par la balle, dans la bulle d'une temporalité autonome, par le fait qu'il se sait évoluer dans un univers virtuel dont il acquiert progressivement la maîtrise (à cet égard, dans le monde enchâssé, sa position est identique à celle du joueur face au monde du jeu); inversement, les jeux Max Payne I et 2 (Remedy Entertainment, 200 I et 2003), portés sur PS2 et Xbox par Rockstar Games plusieurs années avant Red Dead Redemption, naturalisent dans un univers vidéoludique inspiré du genre néo-noir ${ }^{31}$ la vélocité surhumaine du personnage éponyme en intégrant cet effet à leur gameplay. Cette capacité, qui doit nécessairement être limitée dans sa durée d'utilisation - sans quoi l'avatar évoluerait sans résistance aucune dans son environnement, au détriment du pôle de «l'agôn» de l'activité ludique ${ }^{32}-$, porte spécifiquement sur des actions violentes caractérisées par l'usage d'armes à feu.

29 Carl Therrien, «Inspecting Video Game Historiography Through Critical
Lens: Etymology of the First-Person Shooter Genre», Game Studies, vol. I5, $\mathrm{n}^{\circ}$ 2, décembre 20I5. URL: http://game studies.org/1502/articles/therrien.

30 Voir Martin Picard, Pour une esthétique du cinéma transludique: Figures du jeu vidéo et de l'animation dans le cinéma d'effets visuels du tournant $d u X X X I^{e}$ siècle, thèse de doctorat sous la direction de Livia Monnet et Bernard Perron, Université de Montréal, 2009 (non publiée). Disponible en ligne: www.ludov.ca.

31 Il est révélateur que le jeu ait fait l'objet d'une adaptation homonyme au cinéma (Max Payne, John Moore, Canada/ E.-U., 2008), puis, en 20I2, d'un comic book numérique coédité par Rockstar Games et Marvel Comics.

32 Dans la classification de Roger Caillois (Les Jeux et les hommes, Paris, Gallimard, I967 [1958], pp.47-48), «l'agôn» correspond au pôle de la compétition, qui réside ici dans l'interaction entre joueurs (mode multi-joueurs en ligne) ou entre le joueur et la machine (mode histoire). Comme l'a souligné Mathieu Triclot (op.cit., pp. 44-45), les secteurs délimités par Caillois sont à envisager comme des pôles, mobilisés concurremment et à des degrés variables; ainsi le «Bullet Time» des Red Dead relève-t-il aussi de la mimicry (par l'avatar, on y incarne un "cowboy») et de l'ilinx (en raison du vertige produit par l'extension des possibilités perceptives). La durée limitée de l'effet peut se lire également à l'aune d'une polarité d'un niveau supérieur qui traverse selon Caillois tous les types: Red Dead Redemption se donne des airs de paidia (improvisation libre sous la forme d'une déambulation) tout en relevant intrinsèquement du ludus (système de règles). 
33 Alexis Blanchet, Des pixels à Hollywood. Cinéma et jeu vidéo. Une histoire économique et culturelle, Houdan, Pix’n Love, 20Io, p. 99.

$34 \mathrm{Au}$ sens où l'entendait Umberto Eco, Lector in fabula, Paris, Grasset, 1985 [I979], p. I7.

35 En l'occurrence Outlaw (Atari, 1976), Showdown in 2100 A.D. (Duel, Magnavox, I979) et Stampede (Activision, I98I). Notons que le titre original du deuxième jeu, destiné à la console Magnavox Odyssey 2 et sorti cinq ans après le film Westworld (I973), suppose une collusion entre la science-fiction (le «XXI ${ }^{\mathrm{e}}$ siècle» du jeu vidéo) et le monde du western, auquel réfère plus exclusivement la traduction pour d'autres pays (Western pour l'Italie, Pistolero pour les pays hispanophones, Gunfighter en Grande-Bretagne) (voir www.mobygames.com). La description qui en est donnée par Jean-Baptiste Clais est la suivante: «Les personnages sont figurés par de simples blocs de pixels monochromes. Le jeu est en revanche très fluide et ses animations sont conçues de manière à évoquer clairement les gestes et postures de cow-boys. Le jeu joue donc plus sur l'imaginaire des westerns que les joueurs peuvent avoir que sur ses propres graphismes très rudimentaires.» (Game Story. Une histoire des jeux vidéo, Paris, Editions de la RmnGrand Palais, 20II, p. I23).

\section{Jouer au duel: du saloon à la salle d'arcade}

Red Dead Redemption s'inscrit dans la tradition des jeux de tir qui, comme l'illustre avec ironie la séquence inaugurale du film Ride the High Country (Sam Peckinpah, E.-U., I962), remonte à l'attraction foraine. Ce type de jeu d'adresse, rappelons-le, pouvait fonctionner grâce à un système électromécanique, l'usager interagissant avec une forme d'automate. A l'ère de l'informatique, cette pratique ludique se poursuit avec les bornes d'arcade, dont les applications, qui donnent fréquemment lieu à la représentation d'une situation de tir, ont pour la plupart fait l'objet de «portages» sur des consoles de salon. Examinant un vaste corpus de jeux produits entre la fin des années 1970 et I983 par des fabricants tels qu'Atari, Activision ou Mattel, Alexis Blanchet note le rôle prépondérant qu'endossait l'habillage des bornes, dont les motifs dessinés ou photographiques permettaient d'associer une représentation écranique souvent très sommaire (en termes de degré d'iconicité) à un imaginaire (audio)visuel préexistant:

«Les premiers jeux vidéo s’inspirent ainsi des genres cinématographiques ancrés dans l'imaginaire des joueurs et y activent le souvenir d'univers et de conventions, les mécanismes d'un récit rudimentaire, la reconnaissance de répertoires d'action pour l'adoption d'une posture ludique: duel de cowboys, bataille spatiale, fusillade, coursepoursuite, etc.» ${ }^{33}$

Faisant fond sur une certaine familiarité avec le genre du western cinématographique à propos duquel il a acquis des connaissances qui font partie de son «encyclopédie » ${ }^{34}$, le joueur peut ainsi interpréter les représentations schématiques de pixels et se projeter dans une situation vécue par un «cowboy». Blanchet mentionne plusieurs titres de jeux pour le western ${ }^{35}$, et consacre une étude de cas à la cabine du jeu Gun Fight (Midway, I975), dont le système de commande conjugue un joystick pour déplacer l'image du «cowboy» et une crosse de pistolet pour viser et tirer; quant au "péritexte» présent sur la machine, il connote bien sûr l'Ouest américain en s'inspirant, selon Blanchet, à la fois de la série télévisuelle The Wild Wild West (CBS, 1965-1969) et du western spaghetti. L'auteur des Pixels à Hollywood précise à propos de cet horizon référentiel:

«Ce recyclage des codes génériques populaires a pour but d’aider le joueur à adopter une posture ludique grâce à la reconnaissance de réper- 
toires d'action simples et accessibles: se tirer dessus dans un gunfight appartient tout autant au western qu'aux jeux de récréation des petits garçons eux-mêmes calqués sur cet imaginaire cinématographique.» ${ }^{36}$

Le jeu d'adresse s'insère ainsi dans un univers proprement fictionnel, et ses règles propres, parfois dictées par des contraintes techniques, sont naturalisées par la référence à des conventions génériques sur lesquelles repose la vraisemblance des récits (notamment filmiques) ${ }^{37}$. Elles sont implicitement transposées par le spectateur lorsqu'il s'adonne par ailleurs à une activité ludique. Il en va de même dans Red Dead Redemption, dont certains fonctionnements peuvent être jugés problématiques sur le plan de la vraisemblance en ce qu'ils mettent à mal la stabilité mondaine, les développeurs se devant de trouver un équilibre entre d'une part la richesse perceptive et la densité mondaine, d'autre part le confort de jouabilité induit par le taux de rafraîchissement de l'image qui, s'il est élevé, met plus à contribution la machine de calculs. En outre, on constate dans le jeu de Rockstar Games d’inévitables «incohérences» du point de vue diégétique qui résultent de la nécessaire conciliation entre les règles du jeu et le monde fictionnel qu'il propose ${ }^{38}$. Les codifications génériques mettent en quelque sorte de l'huile dans les rouages de cette cohabitation paradoxale en prédéfinissant un horizon d'actions possibles, tout en occultant, inversement, ce que le jeu ne permet pas d'effectuer.

C'est pourquoi l'utilisateur d'une borne comme Gun Fight n'a pas besoin d'être un cinéphile averti ou un lecteur de récits de la conquête de l'Ouest, tant les codes du genre, ainsi que l'indique Blanchet, sont précocement intégrés à cette manifestation d'une "prédisposition anthropologique à l'imitation» que sont les «jeux fictionnels de l'enfance» ${ }^{39}$; or, nous dit Sébastien Kapp, «l'enfant quand il joue utilise des représentations de représentations: son monde imaginaire met fréquemment en scène le monde des adultes, quotidien (jouer au papa et à la maman) ou fictionnel (jouer aux cow-boys et aux indiens)» ${ }^{40}$. Daniel Agacinski observe d'ailleurs dans les westerns que le regard porté sur la figure du gunfighter (sur laquelle nous reviendrons) est systématiquement médié par le regard d'un très jeune protagoniste admiratif ${ }^{41}$. On voit à quel point le «cowboy» fait office, dans la culture populaire, de figure qui excède largement toute référence générique (même s’il en vient souvent à représenter par synecdoque le western) et dont les caractéristiques sont
Alexis Blanchet, op.cit., p. no8.

37 Voir la conception genettienne de la vraisemblance, dans un passage où l'exemple donné relève précisément du western: Gérard Genette, «Vraisemblance et motivation" [I968], dans Figures II, Paris, Seuil, I969, pp. 76-77.

38 Voir à ce propos le chapitre 5 de Jesper Juul, Half-real, op. cit., pp. I63-196, ainsi que la section III.4 de la thèse de Selim Krichane, qui peut ainsi conclure: «Un jeu vidéo peut certes déployer un ensemble de stratégies textuelles et énonciatives afin de «créer un monde`, il peut s'efforcer d'offrir une justification diégétique à l'ensemble des actions entreprises par l'avatar, le «circuit fermé` du calcul informatique n'en restera pas moins présent et apparaîtra nécessairement au joueur» (Selim Krichane, op.cit., p. 365).

39 Jean-Marie Schaeffer, Pourquoi la fiction?, Paris, Seuil, I999, p. 237.

40 Sébastien Kapp, «Jeux de rôles pour enfants: une nouvelle forme de fiction ludique», Strenae, 200I, vol.2, n.p. En ligne: https://halshs.archives-ouvertes. fr/halshs-00741153/document.

41 Daniel Agacinski, "Politique du western: ce que le cinéma fait au mythe», dans Astrid De Munter, Natacha Pfeiffer et Laurent Van Eynde (éd.), Philosophie du western: image, culture, création, Bruxelles, Faculté universitaire SaintLouis, 2012, p. 27. 
42 Dans The Duel (Kieran DarcySmith, E.-U., 20I6), le personnage interprété par Woody Harrelson fait du profit dans une petite ville située à la frontière mexicaine placée sous sa domination en organisant des «visites guidées» pour des touristes qui s'essaient à abattre (réellement) des Mexicains retenus captifs et contraints à servir de cibles dans ce «jeu».

43 Jean-Sébastien Dubé, «New country et nineties' westerns. Parcours parallèles de deux genres en crise d'identité», dans Paul Bleton (éd.), Les Hauts et les bas de l'imaginaire western, Montréal, Tryptique, I997, p. 59.

44 Dubé cite d'ailleurs un article de Janice Rushing qui mentionne également l'activité enfantine: «La rhétorique urbaine du cow-boy est [...] le fun. Il s'agit d'une rhétorique participative - le 〈public ` ne peut être distingué de la source ou du message dans un tel phénomène. Les enfants savent depuis longtemps dissoudre les uns dans les autres ces éléments du processus communicationnel en jouant l'histoire de l'Ouest; aujourd'hui, les adultes semblent avoir accroché à l'idée que jouer cette histoire américaine constitue un excellent moyen de prendre du bon temps» (Janice Hocker Rushing, «The Rhetoric of the American Western Myth», Communication Monographs, $\mathrm{n}^{\circ}$ 50, mars I983, p. 3I, citée dans Jean-Sébastien Dubé, op. cit.).

45 Dans un entretien livré en supplément d'une récente édition de Gli Spezialisti/Le Spécialiste ("Autour du spécialiste», TFI Studio, 20I8), JeanFrançois Rauger souligne cette dimension ludique des westerns de Sergio Corbucci en se référant notamment à la

suffisamment vagues pour s'autonomiser de toute réalité historique ou de tout emprunt à une œuvre spécifique. Cette conception, en phase avec une posture "post-moderne», tend d'ailleurs à se généraliser dans les actualisations récentes d'un genre comme le western, en quelque sorte trop connoté idéologiquement pour être encore pris pleinement au sérieux, d'où la généralisation d'une posture cynique ${ }^{42}$. Ainsi Jean-Sébastien Dubé propose-t-il, en se référant à l'ouvrage Les Jeux et les hommes de Roger Caillois qui a été beaucoup utilisé depuis dans le champ des études vidéoludiques, de «considérer que le country et le western de la dernière vague [c'est-à-dire des années I990] ne prétendent pas tant à maintenir une tradition et un mythe américain, mais bien à <jouer > ce mythe, cette tradition ${ }^{43}$. Les adultes aussi, donc, feraient aujourd'hui l'expérience du western ${ }^{44}$ sur le mode d'une «représentation de représentations»; on peut d'ailleurs prétendre qu'il en allait de même à l'époque de la réalisation des westerns italiens, dont la bouffonnerie des situations dépeintes avec désinvolture érigeait le caractère «ludique» en composante stylistique ${ }^{45}$. On peut dire à cet égard que Red Dead Redemption pousse à son paroxysme la dissolution de la frontière entre création fictionnelle et activité ludique.

\section{Gunfighter/gamer : même combat}

Même si les jeux vidéo, lorsqu'ils ne sont pas présentés comme des adaptations, ne se réfèrent pas explicitement à des productions cinématographiques précises, le type de personnages qu'ils convoquent - ou la "place» à laquelle le joueur est convié à travers les actions à effectuer dans son interaction avec la machine - correspond en général à une période donnée de l'histoire du western cinématographique que l'on peut faire débuter avec un film au titre emblématique, The Gunfighter (Henry King, E.-U., I950), qui se caractérise par un rapport réflexif au genre. Dans un article de i955 consacré à «l'évolution» du western, le critique André Bazin avait en effet perçu cette inflexion dans les productions hollywoodiennes, qualifiant le film Shane (Georges Stevens, E.-U., I953)

séquence finale de I Ragazzi dei Parioli (It., I959) dans laquelle des adultes font mine de tirer sur leurs adversaires avec des pistolets invisibles; pour Rauger, cette scène d'un drame réaliste présagerait le rapport futur du cinéaste au western. 
de «surwestern» ${ }^{46}$. Il n'est donc pas surprenant si c'est précisément de Shane dont se réclamera Sergio Leone au moment de Per un pugno di dollari (1964) ${ }^{47}$, car la tendance repérée en 1955 par Bazin se renforcera au cours de la décennie suivante avec les productions italiennes, pour la plupart teintées d'une grandiloquence parodique. Or tous ces films prennent pour centre de gravité la figure éponyme du film de Henry King: ce sont des gunfighters. Si nous avons jusqu'ici mis entre guillemets le terme «cowboy», c'est qu'il est, dans une perspective historique du moins, impropre à désigner les chasseurs de prime ou hors-la-loi dont les colts crépitent dans tant de westerns, et à propos desquels on peut noter qu'ils ne s'apparentent guère à des vachers utilisant leur pistolet à la seule fin de discipliner un bétail récalcitrant.

Dans l'éclairant chapitre intitulé «Killer Elite» de son ouvrage Gunfighter Nation ${ }^{48}$, Richard Slotkin examine la figure du gunfighter dans des westerns d'après-guerre marqués par un fétichisme de l'arme à feu profondément ancré dans la culture états-unienne, et dont parfois le titre même se résume à la désignation de cet «accessoire» placé au centre de l'intrigue (Colt. 45, Edwin L. Marin, E.-U., I950; Winchester'73, Anthony Mann, E.-U., I950; Springfield Rifle, André de Toth, E.-U., I952; The Gun That Won the West, William Castel, E.-U., I955). Selon Slotkin, le gunfighter constitue une invention du cinéma modelée sur les milieux contemporains du sport et des vedettes de l'écran qui véhicule des stéréotypes de genre associant violence et virilité 49 - ce n'est pas un hasard si Blanchet, dans le passage cité ci-dessus, mentionne les «petits garçons» à propos de la fiction enfantine. Pour ce faire, Slotkin analyse la démarche d'André de Toth qui avait été mandaté par le producteur Darryl F. Zanuck pour écrire une première mouture du scénario de The Gunfighter, et qui prit pour référence un ouvrage composé de biographies d'aventuriers, Triggernometry. A Gallery of Gunfighters d'Eugene Cunningham (I934), soit une source qui adoptait un point de vue très spécifique sur ces figures historiques en soulignant exclusivement,

46 «[... Shane constitue [...] le fin du fin de la "sur-westernisation". En effet Georges Stevens s'y propose de justifier le western par... le western. Les autres s'ingéniaient à faire surgir, des mythes implicites, des thèses fort explicites, mais la thèse de Shane... c'est le mythe. [...] Si le genre western était en voie de disparition, le surwestern exprimerait effectivement sa décadence et son éclatement.
Mais le western est décidément fait d'une autre étoffe que la comédie américaine ou le film policier. Ses avatars n'affectent pas profondément l'existence du genre» («Evolution du western», Les Cahiers du Cinéma, décembre I955, repris dans Qu'est-ce que le cinéma?, Paris, Cerf, I994, p. 233). On peut en dire autant de «l'avatar»(de) Red Dead Redemption.

47 Christopher Frayling, op. cit., p. 27. Pour une comparaison entre le premier western de Leone et Shane, voir Austin Fisher, «Sergio Leone et le western transnational», dans Mary-Dailey Desmarais et Thomas Brent Smith (éd.), Il était une fois le western. Une mythologie entre art et cinéma, Milan, Musée des Beaux-Arts de Montréal/Editions 5 Continents, 2017, pp. 192-I95.

48 Richard Slotkin, Gunfighter Nation. The Myth of the Frontier in TwentiethCentury America, Norman, University of Oklahoma Press, i998. Notons que le terme «Killer Elite» a donné son titre à un film de Peckinpah (qui n'est quant à lui pas un western).

49 Ce que nous exposons ici à propos du gunfighter du western est également valable, mutatis mutandis, pour des figures de soldats. Le film de guerre (ou d'action) constitue en effet une source inépuisable de sujets de jeux vidéo, ces derniers ayant, comme le rappelle Triclot, «dans leur ADN une propension incontrôlable à la «masculinité militarisée`, pour reprendre l'expression de Digital Play» (Mathieu Triclot, op.cit., p. III). Voir Stephen Kline, Nick DyerWitheford et Greg DePeuter, Digital Play. The Interaction of Technology, Culture and Marketing, Montréal, McGill University Press, 2003, p. 246. 
50 Richard Slotkin, op. cit., p. 385 [notre traduction; nous soulignons].

51 Il va de soi que le terme connaît au cinéma des usages préalables. Ainsi un film réalisé par William S. Hart en I9I7 s'intitule-t-il déjà The Gunfighter (significativement traduit en français $L e$ Justicier).

52 Dans The Gunfighter (I950), le personnage éponyme interprété par Gregory Peck est présenté par un carton inaugural qui comporte le texte suivant: «En I88o, dans le Sud-Ouest, la mort et la gloire allaient souvent de pair [was often but a fraction of a second]. La rapidité de leurs réflexes avait fait des héros de Wyatt Earp et Billy the Kid. Mais le tireur le plus rapide était Ringo, un grand gaillard du Texas» (texte du sous-titrage du film, intitulé en français La Cible humaine).

53 Voir le site de The Strong - National Museum of Play: www.museumofplay. org/online-collections/22/67/II2.2922. Dans la séquence liminaire du film Cowboys \& Aliens (Jon Favreau, E.-U., 20II), dans lequel des éléments sciencefictionnels font irruption dans un univers de western, le héros interprété par Daniel Craig se réveille amnésique dans un paysage de l'Ouest américain (aussi «vide» qu'un avatar en début de partie), et découvre qu'il porte un bracelet high tech qui lui permet de repérer l'arrivée d'extraterrestres et de projeter un rayon laser destructeur; l'arme qui fait corps avec lui est à cet égard parente du périphérique de contrôle (d'autant plus à notre époque d'innovation en termes de dispositifs de réalité augmentée) d'un joueur «immergé» dans un environnement de western. parmi la diversité des traits habituellement relevés à leur propos, leur habileté dans le maniement des armes:

«Le livre de Cunningham suggérait que le gunfighting était un métier au même titre que le sport de combat professionnel moderne, un jeu hautement technique, un art noble possédant ses propres règles; il était donc possible d'acquérir une réputation en battant un opposant mieux classé. Par conséquent, être un champion devient alors la marque d’un défi permanent [...].» 50

D’après la définition de Cunningham ${ }^{51}$, dont plusieurs composantes trouvent un écho évident dans des jeux vidéo tels que les Red Dead, le «gunfighter» possède une propriété unique, au sens d'une rareté qui légitime sa position dominante et lui permet d'appartenir à une élite (on parle bien de «tireur d'élite»), mais aussi d'une réduction à une seule compétence que l'on pourrait juger maigre comparativement à la complexité des paramètres intervenant dans la réussite sociale ou la célébrité: la capacité à se distinguer par son habileté au tir ${ }^{52}$. Il importe de relever l'étroite corrélation entre les facultés attribuées de manière stéréotypée à ce type de personnage de fiction et les principes de la jouabilité. Le consommateur de produits vidéoludiques, lui aussi, excelle dans la manipulation d'un artefact prothétique qui prolonge sa main (un périphérique de contrôle), qu'il s'agisse d'un clavier, d'une manette, d'un joystick, ou parfois même, dans le cas de bornes d'arcades, de la crosse d'une arme (Gun Fight), voire d'un pistolet qu'il s'agit de pointer en direction d'un écran ${ }^{53}$. Un duel s'instaure inévitablement entre le joueur et la machine, et la quête du high score peut conduire à une popularité au sein d'une communauté, en particulier dans le cas de jeux en ligne. En schématisant la diversité des pratiques et discours à travers deux pôles d'un continuum de niveaux hiérarchiques, on peut dire en effet qu'il y a l'élite des «true gamers» - ceux par exemple dont les noms apparaissaient parmi les premiers dans les tableaux de score des salles d'arcade, ou de nos jours qui se distinguent comme des pros du e-sport soutenus par des fans qui suivent assidûment leurs exploits sur les plateformes YouTube ou Twitch -, et les autres, c'est-à-dire les joueurs occasionnels. Quel que soit l'univers référentiel d'un jeu vidéo d'adresse, le joueur averti se doit d'être une «fine gâchette». Les jeux vidéo de western transposent mimétiquement cette activité dans la diégèse vidéoludique, naturalisant de la sorte l'adoption des mécaniques de jeu. Il en va de même, il est vrai, 
des jeux dérivés de simulateurs de vol ${ }^{54}$, et, surtout, des très nombreux jeux conférant à l'avatar du joueur l'apparence d'un soldat ${ }^{55}$, c'est-àdire d'une figure qui, dans l'imaginaire nord-américain, s'inscrit dans la filiation du gunfighter ${ }^{56}$. Ce n'est pas un hasard, en effet, si la mise en place dudit stéréotype s'effectue précisément dans le Hollywood d'après-guerre, et si sa généralisation est contemporaine de la guerre du Vietnam. Cette quasi-isomorphie entre gamer et gunfighter nous paraît centrale pour l'examen du statut du western - et plus généralement de tous les genres qui mobilisent la figure d'un tireur - dans les productions vidéoludiques.

\section{Les « grands espaces » d'un monde ouvert}

Dans Les Jeux et les hommes, Roger Caillois considérait déjà la délimitation spatiale comme un élément définitoire de l'occupation ludique ${ }^{57}$. Il y a certes, à un premier niveau, le lieu dans lequel prend place le dispositif (par exemple la pièce dans laquelle se trouvent console et écran), mais aussi, au niveau de la représentation écranique, les espaces traversés par l'avatar ${ }^{58}$. Comme nous l'avons montré ailleurs ${ }^{59}$, la configuration spatiale du monde fictionnel, c'est-à-dire la nature des éléments du monde représenté (portes, murs, ponts, précipices, etc.) - et en particulier leur caractère (in)franchissable -, a des implications décisives sur la jouabilité et sur le type d’immersion proposé au joueur. Jesper Juul souligne que «l'espace dans les jeux peut fonctionner comme une combinaison de règles et de fiction ${ }^{60}$. Cette adéquation entre système de contraintes et possibles narratifs s'opère dans Red Dead Redemption grâce à la coïncidence (tout à fait délibérée) entre les vastes étendues de l’Ouest américain et les caractéristiques d'un «monde ouvert», qui incite le joueur à se

54 D'ailleurs, des films comme The Last Starfighter (Nick Castle, E.-U., I984) ou Ender's Game (Gavin Hood, E.-U., 2013) exploitent sur un plan narratif, et ce jusqu'à la confusion, la similitude entre l'habileté du joueur et les capacités exercées par un combattant dans le monde réel (de la fiction).

55 Mathieu Triclot observe que ce type de jeu est précisément celui qui ambi- tionne de supplanter le cinéma sur le terrain du discours visuel; il mentionne en particulier la reprise "plan à plan», dans le jeu Medal of Honor (Dreamworks, I999), de la scène du débarquement de Saving Private Ryan (Il faut sauver le soldat Ryan, Steven Spielberg, E.-U., I998) sorti l'année précédente sur les écrans et produit par la même firme (M. Triclot, op.cit., pp. 79-80).
56 Voir Richard Slotkin, op. cit., chapitre $\mathrm{I}_{4}$ ("Gunfighters and Green Berets»), pp. 44I-473. Rappelons que de nombreux westerns, à commencer par la célèbre «trilogie de la cavalerie» de John Ford (I948-1950) jusqu'à Hostiles (Scott Cooper, 20I7), ont pour héros un soldat. Il est à ce titre significatif qu'un acteur comme John Wayne qui fut si fortement associé à des rôles de "cowboy" joue parallèlement dans des films de guerre étasuniens réalisés par des cinéastes par ailleurs spécialisés dans le western (They Were Expendable, John Ford, 1945; Sands of Iwo Jima, Allan Dwan, 1949; Operation Pacific, George Waggner, I95I).

57 «Le jeu est essentiellement une occupation séparée, soigneusement isolée du reste de l'existence, et accomplie en général dans des limites précises de temps et de lieu. Il y a un espace du jeu: suivant les cas, la marelle, l'échiquier, le damier, le stade, la piste, la lice, le ring, la scène, l'arène, etc.; mais aussi l'espace représenté» (Roger Caillois, op. cit., p.37).

58 Espen Aarseth postule que les jeux vidéo sont «essentiellement concernés par des représentations et négociations spatiales» (Espen Aarseth, «Allegories of Space», dans Andreas Dieckmann et Peter Russell (éd.), Sauerbraten: The World is a Cube, Bâle, Birkhäuser, 2007, p. I54).

59 Alain Boillat et Selim Krichane, «Les arpenteurs des mondes filmiques et vidéoludiques: pour une théorisation des seuils et des parcours", dans Marc Atallah, Christian Indermühle, Matthieu Pellet et Nicolas Nova (éd.), Pouvoirs des jeux vidéo: des pratiques aux discours, Gollion, Infolio, 20I4, pp. 55-77.

60 Jesper Juul, Half-real, op.cit., p. I63. 
61 Id., pp. 67-83. Pour reprendre la bipartition proposée par Juul, on dira que Red Dead Redemption se situe dans une position médiane entre progression (étapes du récit, missions à accomplir) et émergence (surgissement d'éléments non inscrits dans une chaîne causale). Il arrive fréquemment qu'une mission secondaire se présente de manière impromptue au héros (par exemple l'appel à l'aide d'un personnage croisé en chemin, auquel le joueur peut décider de donner suite ou non). Notons que dans Red Dead Redemption, les éléments sonores jouent un rôle clé dans l'identification (voire dans l'anticipation) d'une action émergente, qu'il s'agisse d'une interpellation vocale par un personnage hors-champ, de coups de feu retentissant dans le lointain ou de la musique extradiégétique qui amorce la dramatisation d'une action à venir.

62 Voir Olivier Mauco, op. cit.

63 Mathieu Triclot, op.cit., p. 62.

64 Selim Krichane, op. cit., pp. I04-105.

65 Id., p. 37.

66 Georges-Albert Astre et AlbertPatrick Hoarau, op. cit., p. 88. déplacer, grâce à divers moyens de locomotion (cheval, train, charrette, diligence, etc.), à travers des paysages qui empruntent au large éventail de la typologie topologique du genre, des plaines désertiques à la Monte Hellmann aux sommets enneigés de Jeremiah Johnson (Sydney Pollack, E.-U., I972), et ainsi à se confronter à des zones qui se présentent comme autant de structures émergentes (au sens de Jesper Juul), de champs d'imprévisibilité ${ }^{61}$. Comme l'exprime Triclot à propos de ce type de jeux, dont Grand Theft Auto (2008), édité par la même firme Rockstar, est un exemple paradigmatique ${ }^{62}$, il s'agit de «donner le sentiment d'un infini à travers le fini, de faire oublier le monde clos du code» ${ }^{63}$. Certes, la «transparence» du monde n'est jamais totale, ainsi que le souligne Selim Krichane en exemplifiant précisément son propos avec Red Dead Redemption:

«Si la remédiation d'une perspective monoculaire [...] favorise un rapport 〈im-média(t) > à l'espace représenté, la figuration en bordure d'écran d'éléments ludiques comme l'arme utilisée, les munitions, ou encore la carte de l'espace de jeu rappellent constamment au joueur qu'il incarne John Marston tout en étant utilisateur du dispositif vidéoludique.» ${ }^{64}$

Il n'en demeure pas moins, comme Krichane l'exprime plus loin, que «la manipulation de la caméra dans Red Dead Redemption (translation $3 \mathrm{D}$ ) participe pleinement du dévoilement continu et progressif de l'espace diégétique qui alimente le plaisir déambulatoire éprouvé par le joueur ${ }^{65}$. Ce plaisir prend un sens tout particulier dans la tradition du western, un genre qui permet, comme l'ont exprimé Astre et Hoarau dans un ouvrage paru l'année de la sortie de Westworld, «de revenir à cette musique du paysage, à cette relation entre l'homme et l'univers, qui à tout prendre nous donne à croire [...] que toute 〈proportionnalité〉 n'est pas détruite entre notre propre personne et un monde qui devient de jour en jour moins commensurable!» ${ }^{66}$. Cette incommensurabilité, que l'on peut rapporter aujourd'hui à la multitude quasi infinie des pages susceptibles d'être traversées par la navigation en ligne, se voit domestiquée par la configuration spatiale de Red Dead Redemption, qui circonscrit une représentation visuelle intégralement numérique dans les trois zones d'un espace de jeu schématisé sous une forme cartographique. Daniel Agacinski note que la répétitivité des décors d'un western à l'autre due au fonctionnement des studios participe à l'unifica- 
tion de l’image symbolique de la Frontière ${ }^{67}$; les éléments programmés, traversés à de nombreuses reprises par l'avatar, jouent un rôle similaire dans Red Dead Redemption. Bien que le monde du jeu soit beaucoup moins meublé et peuplé que celui de séries urbaines telles que Grand Theft Auto (Rockstar Games, dès 1997) ou Assassin's Creed (Ubisoft, dès 2007), et que le temps nécessaire pour rejoindre à cheval le point d'intérêt suivant soit calculé de telle sorte que le jeu puisse concilier impression de vastitude et maintien de l'intérêt du joueur ${ }^{68}$, l'espace vidéoludique est ainsi inscrit dans une mythologie des «grands espaces». Celle-ci est devenue à la fois une imagerie d'Epinal américaine depuis les photographies de grand format réalisées par Carleton E. Watkins ou Timothy O’Sullivan dans le cadre des campagnes d'exploration financées par le gouvernement fédéral dans les années $1870^{69}$, et l'expression métaphorique du cyberspace dans l'imaginaire du cyberpunk ${ }^{70}$. Le sociologue Dominique Cardon décrit ainsi l'idéal des «pionniers» des autoroutes de l'information:

«Internet s'est ainsi donné, comme mythe fondateur, une promesse d'exil et de dépaysement radical. En réanimant l'imaginaire de la Frontière, celui des forêts et des plaines de l'Ouest, le web est apparu à ses fondateurs comme un territoire vierge à conquérir, une contrée indépendante ayant coupé les ponts avec le monde 〈réel `» ${ }^{71}$

Red Dead Redemption superpose l'imaginaire des pionniers de l'Ouest à celui de l'ère informatique. Le type de jeux auquel il appartient est souvent qualifié, en raison du primat de l'espace et de la liberté laissée au joueur, de «bac à sable» (sandbox): quoi de mieux, en effet, que les étendues désertiques du western pour afficher le travail des designers et la puissance des moteurs graphiques? Il pourrait sembler a priori paradoxal de représenter une nature sauvage, dans de nombreux détails de sa flore et de sa faune, grâce à une imagerie entièrement numérique, mais c'est précisément là que réside le défi (identique à celui qui a animé la réalisation de Jurassic Park, un film pensé comme vitrine du potentiel de

67 Daniel Agacinski, op. cit., p. 24.

68 En l'occurrence, le temps de trajet continu (hors les «téléportations» elliptiques motivées diégétiquement par un trajet en diligence) correspond systé- matiquement à une durée jouée de 45 secondes, comme l'a relevé Snaken sur le blog I See What You Did There, n ${ }^{\circ}$. URL: www.gamekult.com/emissions/i-seewhat-you-did-there-8.html. Nos remer- ciements à Jérôme Jacquin pour cette référence. L'analyse proposée par Snaken montre que le temps pré-calculé qui participe à la définition de la topographie de l'espace interne au jeu est strictement identique dans d'autres productions vidéoludiques à monde ouvert, de sorte qu'il s'agit là d'une sorte de standard. Il va de soi toutefois que cette caractéristique quantitative est établie à partir d'un déplacement effectué à vitesse maximale, tandis que le joueur peut tout à fait décider d'être moins « efficient» et plus contemplatif en prenant le temps d'exploiter la malléabilité de son environnement.

69 Voir François Brunet et Bronwyn Griffith (éd.), Visions de l'Ouest. Photographies de l'exploration américaine, I860-I87o, Chicago/Paris, Terra Foundation for American Art/RMN, 2007.

70 Rappelons à cet égard que le romancier William Gibson qualifiait de «cowboy» son héros, un hacker hors-la-loi: «Case avait vingt-quatre ans. A vingtdeux, il était un cow-boy, un braqueur, l'un des tout bons de Zone. Sa formation, il la tenait des meilleurs, les McCoy Pauley et autres Bobby Quine, des légendes dans le métier. Il avait pratiqué un trip d'adrénaline pratiquement permanent, un sous-produit de la jeunesse et de la compétence, branché sur une platine de cyberspace maison qui projetait sa conscience désincarnée au sein de l'hallucination consensuelle qu'était la matrice» (William Gibson, Neuromancier, Paris, J'ai Lu, 20Iо [Neuromancer, I984], p. 8).

71 Dominique Cardon, La Démocratie Internet. Promesses et limites, Paris, Seuil, 20I0, p. 25. 
72 Geoff King et Tanya Krzywinska, Tomb Raiders \& Space Invaders. Videogame Forms \& Contexts, Londres/New York, Tauris, 2006, pp. 76-I23.

73 Geoff King et Tanya Krzywinska, op. cit., p. 98.

74 Le joueur dispose non seulement d'un indicateur de géolocalisation, mais aussi d'une carte lui permettant de se repérer et d'effectuer des déplacements rapides d'un point à un autre: s'il décide d'installer un campement en un lieu donné, il peut ensuite poser un repère sur la carte, et ainsi bénéficier d'une élision de la représentation du déplacement. L'espace peut être également balisé par le joueur grâce à des repères particuliers qui, dans la diégèse, correspondent à des habitations louées ou achetées.

75 Parmi les exemples de «limites malléables» [soft boundaries] mentionnées par King et Krzywinska (où la limitation correspond souvent à son incarnation spatiale dans une «frontière»), le pont constitue un motif récurrent. Voir leur commentaire à propos des jeux Grand Theft Auto III (Rockstar Games, 200I) et The Getaway (Team Soho, 2002). Geoff King et Tanya Krzywinska, op. cit., p. 95.

76 Les conventions sociales consistent par exemple en l'interdiction de camper à proximité d'une ville, ou en la fermeture des magasins la nuit. En ce qui concerne la justification de l'inaccessibilité de certains espaces, on peut s'étonner, du point de vue de la vraisemblance (c'est-à-dire de la motivation diégétique des règles du jeu), de l'inaptitude complète de John Marston à la nage, tout contact avec une zone aquatique provo-

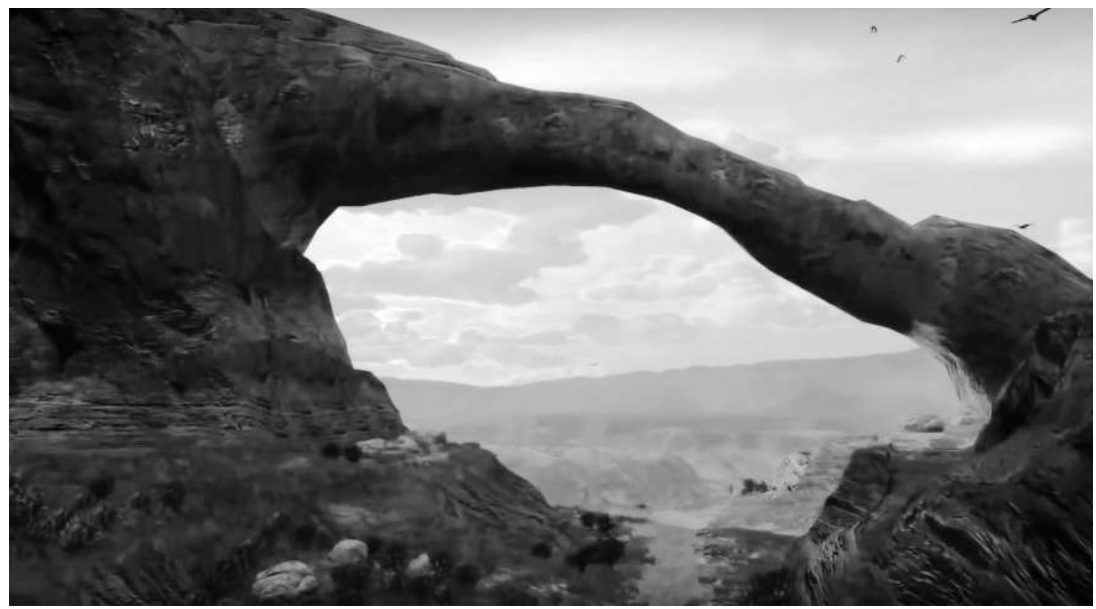

19/ Silhouettes rocheuses baignées dans la lumière de l'aube: image tirée de Red Dead Redemption

l'infographie). L'image au format panorama du menu de Red Dead Redemption, qui rappelle les films en Scope, annonce la couleur. Le temps diégétique étant régi par un cycle jour/nuit progressif, les variations de luminosité au cours de la «journée» sont rendues avec une qualité qui exacerbe la «picturalité» de la représentation - voir par exemple la référence au parc national des Arches (fig. I9).

Dans leur chapitre intitulé «Gamescapes», Geoff King et Tanya Krzywinska ${ }^{72}$ établissent pour l'ensemble de la production vidéoludique des critères d'analyse de l'espace. Si nous les appliquons à Red Dead Redemption, on observe une maximisation de l'ensemble des paramètres mentionnés, que cela soit au plan du degré de présence, renforcé selon les auteurs dans les «jeux pleinement tridimensionnels à la troisième personne ${ }^{73}$ tels que Red Dead Redemption, ou au plan du degré de liberté laissé au joueur dans sa découverte du monde. En effet, le gameplay du jeu encourage l'exploration ${ }^{74}$, et cela d'autant plus après l'ouverture de l'espace de jeu qui se concrétise, à partir d'un certain degré de progression, par la reconstruction d'un pont donnant accès au Mexique ${ }^{75}$. Quant aux contraintes ludiques, elles sont en général naturalisées par la référence à des habitus sociaux, ou par la nature du terrain ${ }^{76}$. 


\section{La possibilité d'un paysage}

Une affiche figurant parmi le matériel d'exploitation du diffuseur japonais Capcom pour le jeu Mad Dog McCree (I990) ${ }^{77}$ développé par American Laser Games (fig. 20) met l'accent sur l'environnement présenté dans l'image photographique de Monument Valley à l'arrière-plan, c'est-à-dire un espace à l'imagerie préconstruite ${ }^{78}$, un parc national ${ }^{79}$ navajo nettement délimité dont l'entrée est payante et qui comprend un repère à l'endroit d'où John Ford filma ses plus célèbres plans («John Ford Point»); or ce panorama est en partie dissimulé par l'écran du jeu qui, en

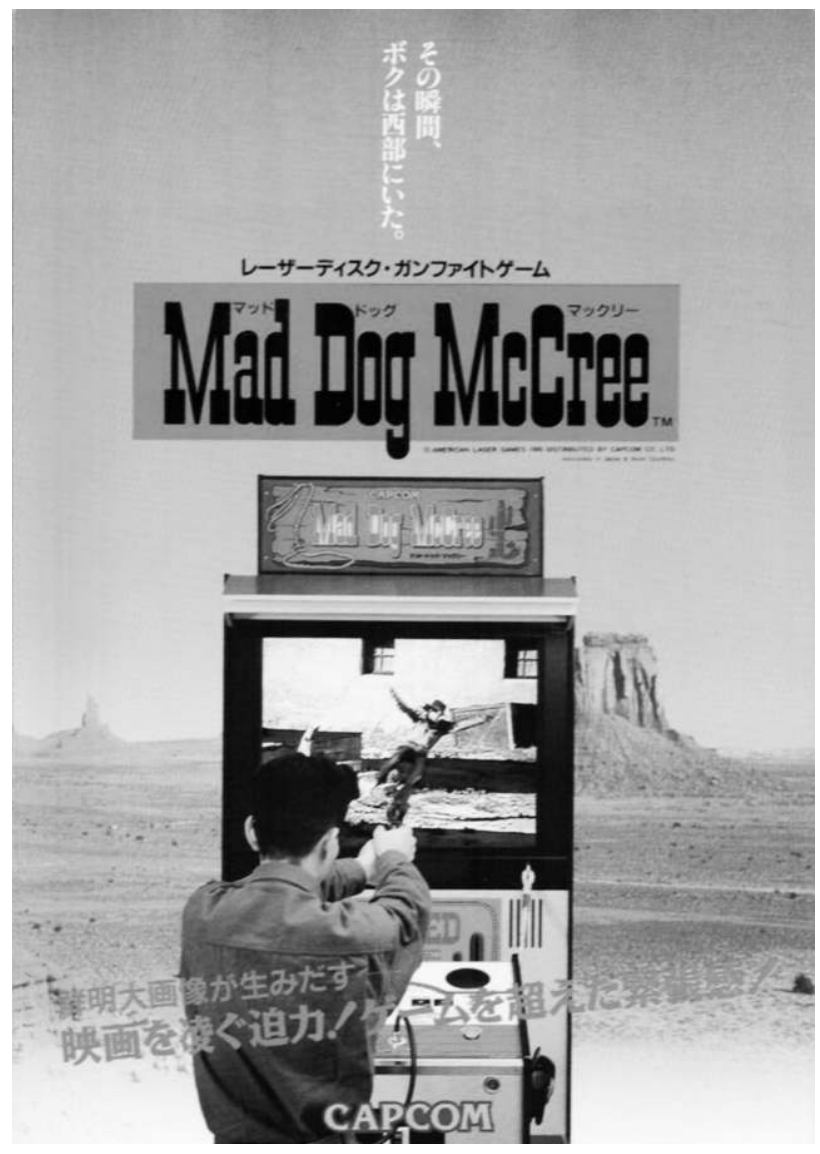

77 Les réclames et modes d'emploi sont disponibles sur le site GameTronik (www. gametronik.com/site/fiche/daphne/Mad $\% 20$ Dog\%20McCree/). Les images animées sont intégrées dans un dispositif interactif et ludique, puisque le jeu propose une image filmée en prise de vues réelles stockée sur un lecteur de disques laser Sony (avec un second disque pour le son). C'est ce qui explique le choix de Janet Murray d'exemplifier avec ce jeu une généalogie postulée entre cinéma et jeux vidéo, ainsi que les «objectifs» du médium en termes de narration et d'immersion (Janet Murray, Hamlet on the Holodeck. The Future of Narrative in Cyberspace, Cambridge, MIT Press, 1998 [1997], pp.54-55; pour une étude du discours de Murray à propos de ce jeu, voir Selim Krichane, op. cit., pp. 88-9I).

78 Il ne s'agit donc pas, dans le cas de Red Dead Redemption, d'opposer un réel à une représentation produite par une machine informatique, dans la mesure où les paysages de l'Ouest relèvent eux-mêmes d'une imagerie à la facticité intrinsèque.

79 La notion de "parc national» constitue l'un des modèles de l'espace diégétique de Red Dead Redemption. A propos de l'aménagement en 1876 du premier parc américain, Yosemite, voir François Brunet et Bronwyn Griffith (éd.), op. cit.; les auteurs le qualifient significativement de «sanctuaire naturel [...] envisagé comme une arène des plaisirs visuels » (p. 15).
20/ Publicité pour Mad Dog McCree (1990): enchâssement du film dans le jeu, et du jeu dans le décor 
21/ Le héros interprété par James Stewart dans Bend of the River (Les Affameurs, Anthony Mann, E.-U., 1952) regarde s'éloigner la caravane, non le paysage qui pourtant s'impose à l'arrière-plan

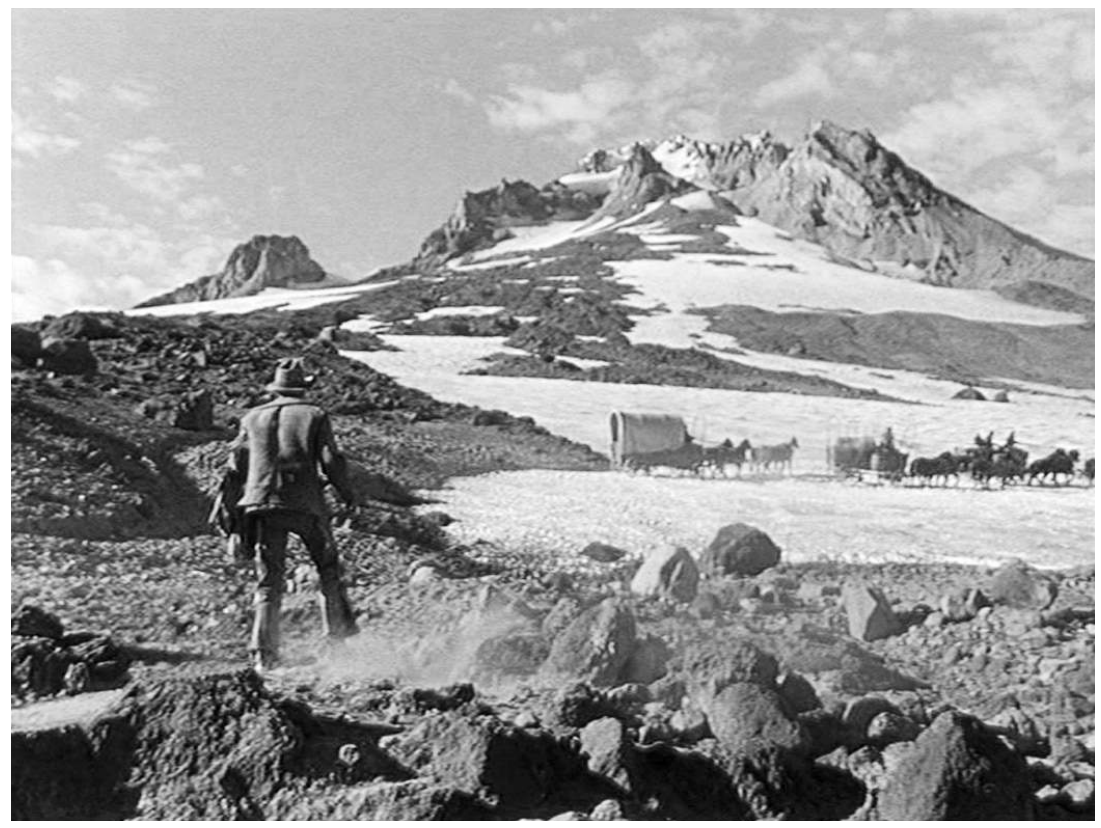

80 Nos remerciements à David Javet pour sa traduction du japonais.

81 Geoff King et Tanya Krzywinska, op. cit., p. 76 .

82 Il est à cet égard intéressant de mentionner l'étude dans laquelle Martha Sandweiss montre que les éditeurs ont préféré des gravures aux daguerréotypes pour illustrer les ouvrages d'exploration, car les premières permettaient mieux d'adapter la représentation au récit épique; les images numériques de Red Dead Redemption constituent le paroxysme d'une telle emprise sur l'image. Martha A. Sandweiss, Print the Legend. Photography and the American West, New Haven/Londres, Yale University Press, 2002, p. 97 . quelque sorte, se substitue à lui. Le texte qui apparaît à la verticale dans la partie supérieure de la publicité pose une affirmation à destination du public japonais qui fait office de promesse de dépaysement et de récits: "A cet instant, j'étais dans l'Ouest» ${ }^{80}$. Grâce au monde ouvert qu'il propose, Red Dead Redemption amplifie considérablement ce sentiment de présence, d'inscription dans un espace, se distinguant ainsi grandement des shoot'em up centrés sur la seule action. King et Krzywinska mentionnent la possibilité pour certains jeux vidéo «d'inclure l'objectif de se déplacer librement à l'intérieur et au travers d'une diversité de paysages qui apparaissent dans le champ, un plaisir qui peut susciter un attrait pour lui-même» ${ }^{81}$. L'autonomisation potentielle de paysages en des images de cartes postales ${ }^{82}$ livrées à la contemplation du joueur constitue une caractéristique essentielle du jeu, qui prend une signification toute particulière en regard des attentes génériques. Certes, comme l'a affirmé Triclot, «une image de jeu vidéo est destinée à être scannée à la recherche d'éléments actionnables, non à être regardée dans sa globalité 
comme une photographie ${ }^{83}$. Toutefois, à ce titre, Red Dead Redemption fait selon nous figure d'exception en s'inscrivant dans un genre cinématographique qui est constamment discuté pour l'importance conférée aux paysages (que ce genre a précisément contribué à établir dans sa valeur esthétique) ${ }^{84}$ tout en n'offrant que très rarement des instants de contemplation échappant à toute visée actionnelle - si les personnages des films scrutent leur environnement, c'est parce qu'ils sont constamment sur le qui-vive, aux aguets, instaurant un rapport utilitariste à un espace que le récit les oblige à maîtriser. Alors que l'avatar de Red Dead Redemption peut rester inactif et contempler le paysage - les sollicitations de la machine à coup de raccords imposés sur une partie spécifique du paysage prévue en tant que zone d'émergence cessent après quelques minutes -, c'est en fait, dans le western cinématographique, le spectateur qui profite du paysage dans lequel s'inscrit le personnage, mais au détriment (ou comme à l'insu) de celui-ci (fig. 2I). D’ailleurs, Maureen Turim a noté à propos de la «vision du paysage, grandiose et texturé, emblématique des grands espaces qui s'offrent au spectateur» que «ce type de plan est plus rare dans le western qu'on ne le pense généralement ${ }^{85}$. Peut-être même, d'ailleurs, n'existe-t-il pas à proprement parler, si ce n'est dans un jeu à monde ouvert tel que Red Dead Redemption, qui offre entre autres la possibilité d'actualiser pleinement un fantasme... de cinéma.

Parmi les nombreuses activités ou missions qui s'offrent à John Marston, on trouve la visite d'une salle de cinéma où l'avatar peut assister à la projection d'un film. Cette «action» emblématise la fusion du «cowboy» et du spectateur de manière littérale. Si le film auquel «nous» (c'est-à-dire la «trinité» joueur/avatar/personnage) assistons est un court-métrage d'animation en noir et blanc, c'est que les concepteurs de Red Dead Redemption biaisent la comparaison avec le médium cinématographique en l'identifiant à un mode de représentation beaucoup moins "photoréaliste» que celui proposé par le jeu - même si le dessin s'inscrit plus spécifiquement dans la généalogie de l’image générée informatiquement ${ }^{86}$. Lors de la séance de cinéma interne à la diégèse vidéoludique, les capacités de la «caméra» du jeu à la troisième personne sont d'ailleurs désactivées au profit de la possibilité réduite d'un raccord dans l'axe ( fig. 2223), figure de montage qui est elle-même caractéristique du cinéma des premiers temps. De là à induire, à l'instar des discours promotionnels
83 Mathieu Triclot, op.cit., p. 95.

84 Voir l'exemple de la Monument Valley chez John Ford, discuté par Suzanne Liandrat-Guigues et Jean-Louis Leutrat, Splendeur du western, Pertuis, Rouge Profond, 2007, pp. 77-83.

85 Maureen Turim, «L'encadrement de paysage», dans Gilles Menegaldo et Lauric Guillard (éd.), Le Western et les mythes de l'Ouest. Littérature et arts de l'image, Rennes, PUR, 2005, p. 395.

86 Voir André Gaudreault et Philippe Marion, La Fin du cinéma?, Paris, Armand Colin, 2013, chapitre 7 («<L'animage > et la nouvelle culture visuelle»), pp. 2 II -248 . 
Activant un «zoom», le joueur de Red Dead Redemption devient spectateur: présence explicite du cinéma sous la forme schématisée d'un dessin animé humoristique de western projeté à proximité du drapeau américain
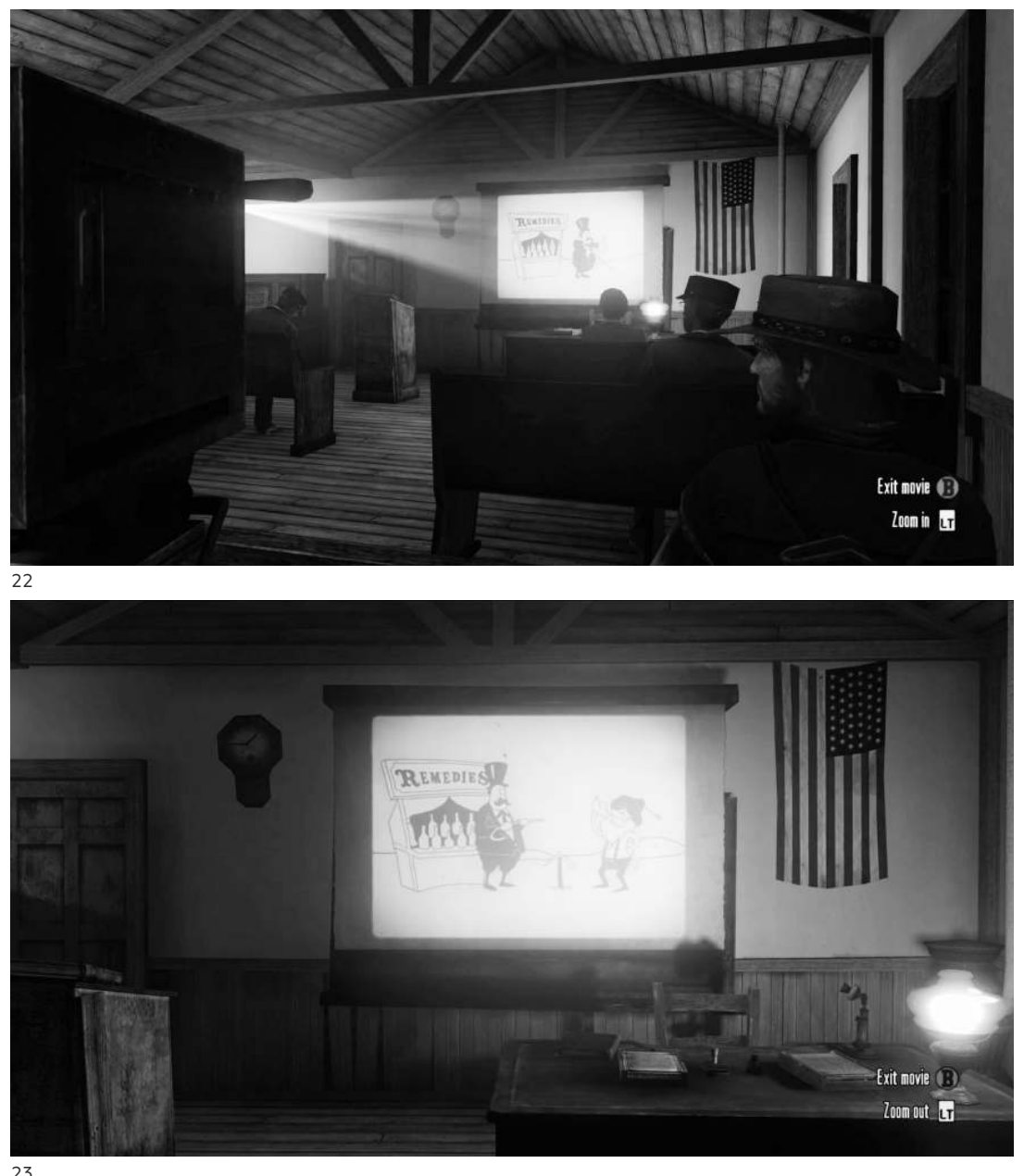

23

87 Ainsi, le texte figurant dans la partie inférieure de la promotion japonaise pour Mad Dog McCree (fig. 2o) est la suivante: "Une grande image nette est créée. Un impact qui éclipse le cinéma! Une tension qui a surpassé le jeu!» [trad. David Javet]. usuels de l'industrie du jeu vidéo ${ }^{87}$, que c'est le cinéma lui-même, grand pourvoyeur d'imageries westerniennes, qui, sur le terrain de la contemplation des paysages de l'Ouest, est devenu anachronique avec le succès de Red Dead Redemption, il n'y a qu'un pas... 